\title{
Ethylene Oxidation Chemistry in a Well-Stirred Reactor
}

\author{
Nick Marinov and Phil Malte \\ University of California \\ Lawrence Livermore National Laboratory \\ Livermore, CA 94550
}

September 1994

This is an informal report intended primarily for internal or limited external distribution. The opinions and conclusions stated are those of the author and may or may not be those of the Laboratory.

Wogk performed under the auspices of the U.S. Department of Energy by the Lawrence Livermore National Laboratory under Contract W-7405-Eng-48.

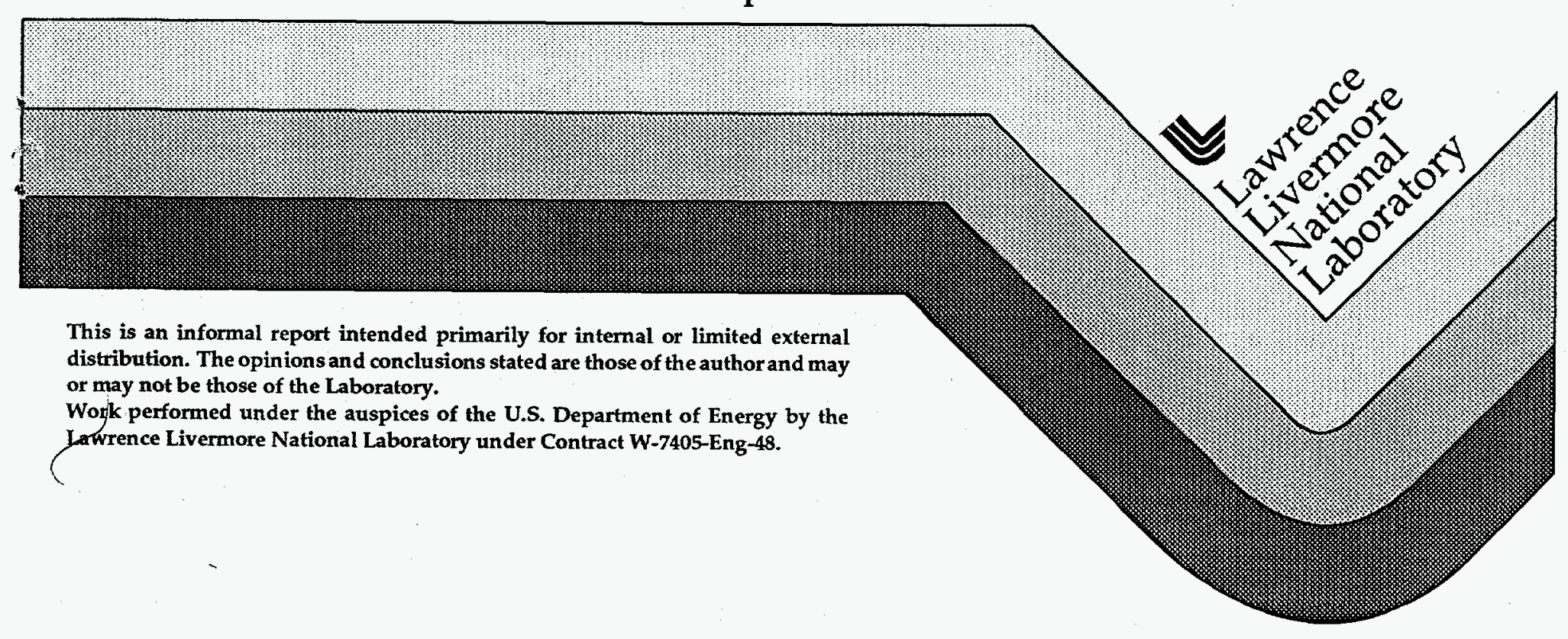




\section{DISCLAIMER}

This report was prepared as an account of work sponsored by an agency of the United States Government. Neither the United States Government nor any agency thereof, nor any of their employees, make any warranty, express or implied, or assumes any legal liability or responsibility for the accuracy, completeness, or usefulness of any information, apparatus, product, or process disclosed, or represents that its use would not infringe privately owned rights. Reference herein to any specific commercial product, process, or service by trade name, trademark, manufacturer, or otherwise does not necessarily constitute or imply its endorsement, recommendation, or favoring by the United States Government or any agency thereof. The views and opinions of authors expressed herein do not necessarily state or reflect those of the United States Government or any agency thereof. 


\section{DISCLAIMER}

Portions of this document may be illegible in electronic image products. Images are produced from the best available original document. 


\title{
ETHYLENE OXIDATION CHEMISTRY IN A WELL-STIRRED REACTOR
}

\author{
Nick Marinov \\ Lawrence Livermore National Laboratory \\ P.O. Box 808 \\ Mail Stop: L -298 \\ Livermore, California 94551 \\ and \\ Phil Malte \\ Department Of Mechanical Engineering \\ University of Washington \\ Mail Stop: FU - 10 \\ Seattle, Washington 98195
}

Correspondence should be addressed to:

Nick Marinov

Lawrence Livermore National Laboratory

P.O. Box 808

Mail Stop: L -298

Livermore, California 94551

Phone: (510) 424-5463

Fax: (510) 422-2851

Internet E-Mail: marinov@llnl.gov 


\title{
ETHYLENE OXIDATION CHEMISTRY IN A WELL-STIRRED REACTOR
}

\begin{abstract}
Detailed fuel-lean $\mathrm{C} 2 \mathrm{H} 4 / \mathrm{H} 2 \mathrm{O} / \mathrm{AIR}$ well-stirred reactor data by Thornton [1] were used to analyze reported combustion chemistry mechanisms and the development of this study's ethylene oxidation mechanism. The data set had been obtained for the temperature range 1003 to $1253 \mathrm{~K}$ and ethylene-oxygen equivalence ratio range 0.086 to 0.103 , at atmospheric pressure. Mechanisms were derived from reaction sets of Westbrook and Pitz [2], and Dagaut, Cathonnet and Boettner [3]. Examination of each reported mechanism indicated unusually large kinetic rates for the vinyl decomposition reaction were used in order to obtain agreement with the Thornton data set.
\end{abstract}

An ethylene oxidation model was developed in order to address the mechanistic problems of the previous models. This study's mechanism well simulated the overall rate of ethylene oxidation and concentation profiles of $\mathrm{CO}, \mathrm{CO} 2, \mathrm{H} 2, \mathrm{CH} 2 \mathrm{O}, \mathrm{C} 2 \mathrm{H} 2, \mathrm{CH} 3 \mathrm{OH}, \mathrm{CH} 4$, and $\mathrm{C} 2 \mathrm{H} 6$. Successful predictions by the model were dependent on a new high temperature vinyl oxidation reaction route,

$$
\mathrm{C} 2 \mathrm{H} 3+\mathrm{O} 2=\mathrm{CH} 2 \mathrm{CHO}+\mathrm{O}
$$

with a $\mathrm{k}_{\mathrm{C}} 2 \mathrm{H} 3+\mathrm{O} 2=\mathrm{CH} 2 \mathrm{CHO}+\mathrm{O} / \mathrm{k}_{\mathrm{C}} 2 \mathrm{H} 3+\mathrm{O} 2=\mathrm{CH} 2 \mathrm{O}+\mathrm{HCO}$ branching ratio of 1.19 -1.21 at $1053 \mathrm{~K}$ to $1.63-2.47$ at $1253 \mathrm{~K}$. The branching ratio values were dependent upon the extent of fall-off for the $\mathrm{C} 2 \mathrm{H} 3+\mathrm{O} 2=\mathrm{CH} 2 \mathrm{O}+\mathrm{HCO}$ reaction. 


\section{INTRODUCTION}

Ethylene is an important intermediate in the combustion of methane [4,5], larger aliphatic hydrocarbons [6,7], and aromatics [8]. Consequently, its oxidation is considered to be one of the principal submechanisms for the development of kinetic models for complex fuels. We have identified nine studies devoted to the modeling of ethylene oxidation [2,9-16]. Experimental data used to validate these models were obtained from flow, static and jet-stirred reactors and from shock tubes and flames. The experimental results used in this study to test existing $\mathrm{C}_{2}$ mechanisms and the presently compiled model were obtained by Thornton [1] using a well-stirred reactor operated at atmospheric pressure, for the temperature range 1003 to $1253 \mathrm{~K}$ and ethylene-oxygen equivalence ratio of 0.086 to 0.103 . Initial modeling of these results was undertaken and reported by Westbrook et al. [2] and includes Thornton's complete data set.

That work reported that $\mathrm{H}$-atom abstraction from ethylene by $\mathrm{OH}$ attack dominated fuel consumption. The rate expression due to Tully [17] represented this primary oxidation pathway and reproduced the wellstirred reactor experimental observations for the loss of ethylene in the 1150 to $1200 \mathrm{~K}$ range. However, the experimental results at other temperatures were not well simulated by the model, nor were the measured intermediate species well simulated.

In the present study, reported chemical kinetic mechanisms of Westbrook/Pitz (WP) [2], and Dagaut/Cathonnet/Boettner (DCB) [3] were examined by comparing predicted species concentration profiles against experimental measurements of Thornton. These mechanisms were previously tested against the Thornton data set and served as a starting point in the present investigation. Sensitivity and reaction pathway analysis were used to identify of the important reactions controlling the ethylene oxidation process and intermediate concentrations. Differences in the predictions amongst the 
mechanisms were determined and discussed in terms of reaction pathways and/or rate expressions used. After a detailed examination of each reported mechanism was conducted an ethylene oxidation model was developed to address the inadequacies represented by the existing mechanisms.

\section{EXPERIMENTAL - DATA SOURCE}

The data of Thornton [1] were obtained in a well-stirred reactor using gas chromatography. The details of the well-stirred reactor have been published previously [18,19], including verification of the wellstirred behavior. The stirred reactor was operated at one atmosphere, temperature range of 1003 to $1253 \mathrm{~K}$, nominal residence time of $2 \mathrm{msec}$, and ethylene-oxygen equivalence ratio range of 0.086 to 0.103 . The inlet composition consisted of complete products of very fuel-lean hydrogen combustion of approximately $11 \% \quad 02,14 \% \quad \mathrm{H} 2 \mathrm{O}$, and $75 \% \quad \mathrm{~N} 2$ together with the amount of ethylene (0.36\%) seeded into the flow. All values are molar.

Detailed multipoint calibration curves were generated for $\mathrm{H} 2, \mathrm{CO}, \mathrm{CO} 2$, $\mathrm{O} 2$, and $\mathrm{C} 2 \mathrm{H} 4$, where there was concern about qualitative accuracy over the range of concentrations seen in these experiments. Only in rare cases was the accuracy in the concentration measurements poorer than that of the calibration standards of $2 \%$. Thornton determined the uncertainties to the measured species concentration to be approximately $2 \%$ for $\mathrm{CH} 4$ and $\mathrm{C} 2 \mathrm{H} 6$; $10 \%$ for $\mathrm{C} 2 \mathrm{H} 2$; and $20 \%$ for $\mathrm{CH} 2 \mathrm{O}$ and $\mathrm{CH} 3 \mathrm{OH}$. No measurements were undertaken for free radicals. Errors introduced by jet-stirred reactor inhomogeneity were considered to apply only to components derived from the introduction of the ethylene additive. Measurement uncertainties due to reactor inhomogeneity are estimated to be $40 \%$ for $\mathrm{CH} 2 \mathrm{O}$ and $\mathrm{CH} 3 \mathrm{OH}$, and an estimated uncertainty of $20 \%$ for all other species. An elemental carbon and oxygen balance was checked at all temperatures and was found good to $101 \pm 1.0 \%$ and $102 \pm 1.6 \%$ respectively. 
Non-catalytic coated type $R$ platinum-rhodium thermocouples were used to measure the reactor temperature. Uncertainties from radiation and conduction losses are estimated to be less than $20 \mathrm{~K}$.

\section{COMPUTATIONAL MODEL AND MECHANISMS}

The computational model used for this study is the Sandia micromixed perfectly stirred reactor (PSR) code [20]. The model considers the $15.8 \mathrm{cc}$ reactor volume in which the experiments took place. Input parameters are the measured reactor temperature, inlet mass flow rate, and inlet composition.

The reported mechanisms considered in this study are summarized below:

1. The mechanism of Westbrook and Pitz (WP) was initially developed to analyze the experimental results of ethylene oxidation by Thornton. The detailed numerical modeling analyses has been reported, however, a reexamination of their mechanism was conducted for the purposes of identifying which reaction or interacting set of reactions cause agreement or disagreement between the full data set and predictions. Both forward and reverse rates are given in the mechanism, thus insuring use of their thermochemistry.

2. The Dagaut, Cathonnet and Boettner (DCB) mechanism is an improved version of their previous ethylene reaction sets [15,16]. This particular mechanism had been validated for ethylene, methane and ethane combustion for the conditions of 1-10 atmospheres, 900-1200K temperature range and fuel-air equivalence ratio range 0.1-1.5. Sixteen unimolecular and bimolecular chemically activated reactions have been pressure corrected to one atmosphere and are included in the mechanism. Only the forward rate expressions were cited and thermodynamics were taken from the Chemkin Thermodynamics Database [21] and Burcat thermochemical data (i.e. $\mathrm{C} 2 \mathrm{H} 4 \mathrm{O}$ ) [22]. 
While these kinetic schemes may not represent the current mechanisms of these authors, the aim of the present analysis is to elucidate the important reactions controlling the ethylene oxidation process and reaction intermediates formed from therein.

\section{RESULTS OF THE REPORTED MECHANISMS}

Concentration profiles for the measured stable species (C2H4, CO, $\mathrm{CO}$, $\mathrm{H} 2, \mathrm{CH} 2 \mathrm{O}, \mathrm{C} 2 \mathrm{H} 2, \mathrm{CH} 3 \mathrm{OH}, \mathrm{CH} 4$, and $\mathrm{C} 2 \mathrm{H} 6$ ) as computed using the WP and DCB mechanisms have been compared with the Thornton data set. The full presentation of the numerical computations and discussion are found in Marinov [23]. Only a summary of the important findings are presented here.

The two mechanisms showed good agreement for the loss of ethylene in only a limited temperature range. The WP mechanism well represented the loss of ethylene for only the $1150-1200 \mathrm{~K}$ temperature range. The DCB mechanism showed a better representation for the loss of ethylene over the $1050-1200 \mathrm{~K}$ temperature range. Reaction pathway analysis indicated that approximately $40-50 \%$ of the ethylene is consumed by $\mathrm{OH}$ radicals followed by a substantial secondary amount of consumption by $\mathrm{O}$-atom. In contrast, alkyne flame modeling studies [24] found oxidation by $\mathrm{O}$-atom to be critical in fuel-lean to fuel-rich lightly sooting flame; while in alkane flames, oxidation by $\mathrm{OH}$ and $\mathrm{H}$-atom at fuel-lean to stoichiometric conditions are considered to be important.

Intermediates, in general, were not well simulated by the WP mechanism while DCB simulated all stable species, except for acetylene and methanol, fairly well for temperatures up to $1200 \mathrm{~K}$. Acetylene was overpredicted by a factor of 20 to 30 for both mechanisms and methanol was underpredicted by approximately a factor of 10 by DCB. DCB overpredicted ethane by a factor of two to three for the $1050-1200 \mathrm{~K}$ temperature range, while in WP, ethane was underpredicted by roughly 
one order of magnitude. The two mechanisms predicted formaldehyde to within a factor of two for all temperatures. Methane, hydrogen, carbon monoxide and carbon dioxide species were well-simulated by DCB for temperatures to $1200 \mathrm{~K}$, while WP simulated these species well only around $1178 \mathrm{~K}$. Figures 1 and 2 show the measured and predicted profiles by WP and DCB for ethylene, acetylene and methanol.

Temperatures greater than $1200 \mathrm{~K}$, the loss of ethylene is overpredicted by both mechanisms. Poor mixing of the gases at the microscale was suggested by WP and $D C B$ as the reason for the observed disagreement between model and experimental measurement.

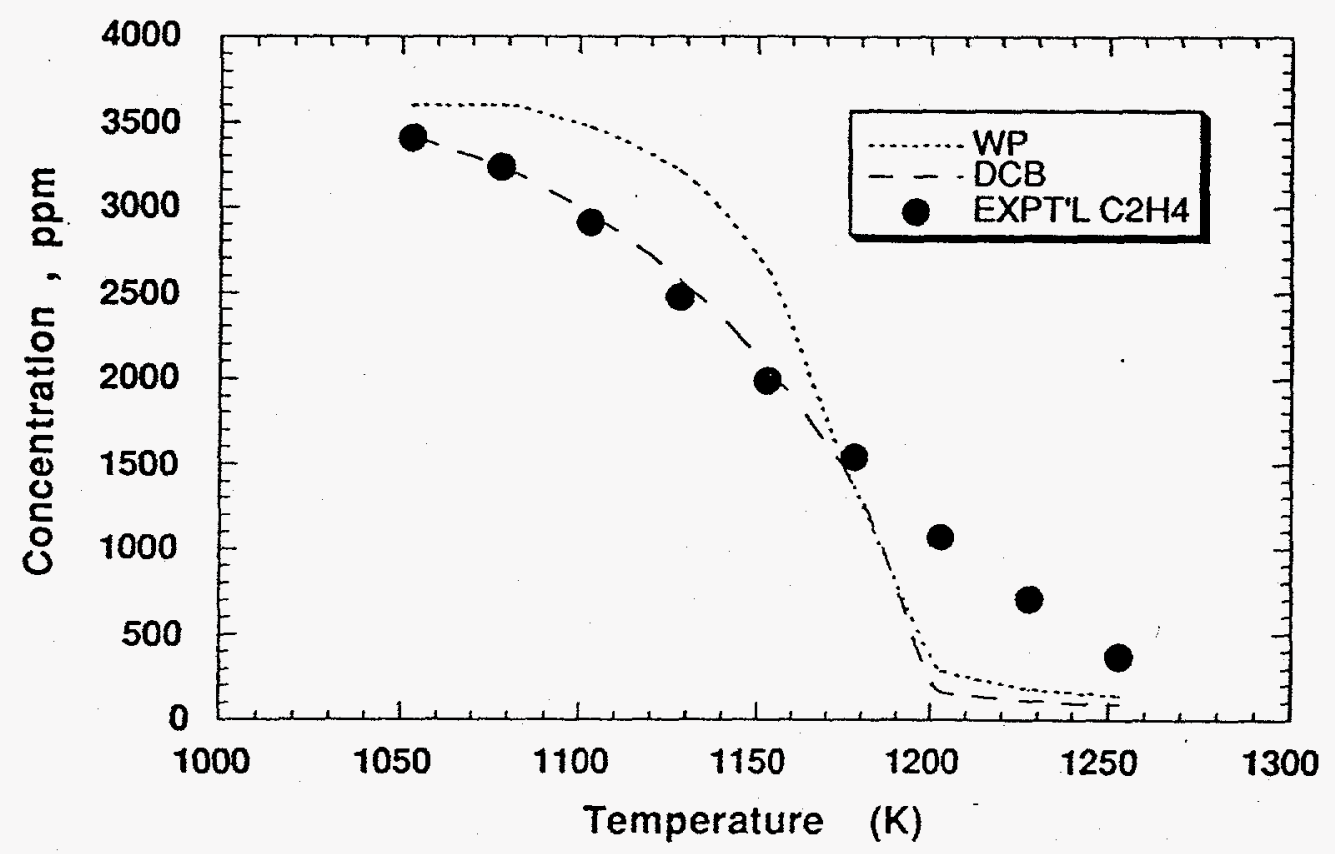

Figure 1: Comparison of predicted and experimental concentration profile of ethylene in the well-stirred reactor for ethylene oxidation: Experimental (solid symbols), from Thornton; predicted curves, from the mechanisms of Westbrook and Pitz (WP); and Dagaut, Cathonnet, and Boettner (DCB). 


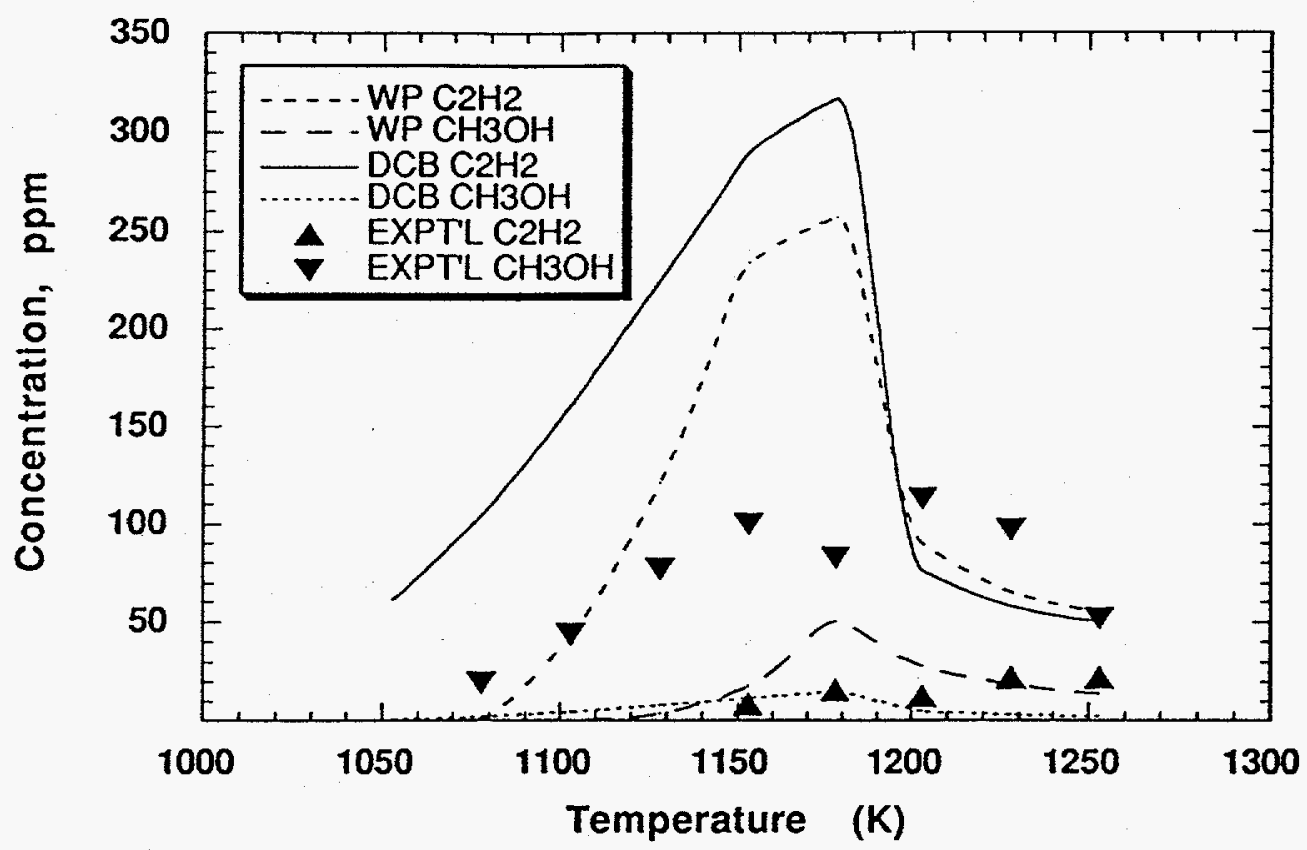

Figure 2: Comparison of predicted and experimental concentration profiles of acetylene and methanol in the well-stirred reactor for ethylene oxidation: Experimental (solid symbols), from Thornton; predicted curves, from the mechanisms of Westbrook and Pitz (WP); and Dagaut, Cathonnet, and Boettner (DCB).

The principal difference in the model predictions between the DCB and WP mechanisms lies in the kinetic rate choice for the vinyl decomposition reaction. $\mathrm{DCB}$ used a two orders of magnitude faster vinyl decomposition rate than WP which allowed vinyl decomposition to predominate over vinyl oxidation by $\mathrm{O} 2$. The early production of $\mathrm{H}$-atom from $\mathrm{C} 2 \mathrm{H} 3=\mathrm{C} 2 \mathrm{H} 2+\mathrm{H}$ allows for rapid chain branching through $\mathrm{H}+\mathrm{O} 2=$ $\mathrm{OH}+\mathrm{O}$. The formed $\mathrm{O}$-atom will preferentially react with $\mathrm{C} 2 \mathrm{H} 4$ producing $\mathrm{CH} 3+\mathrm{HCO}$ and $\mathrm{CH} 2+\mathrm{HCO}+\mathrm{H}$ in $\mathrm{DCB}$ or $\mathrm{CH} 3 \mathrm{CO}+\mathrm{H}$ in WP. The $\mathrm{H}$-atom produced from $\mathrm{C} 2 \mathrm{H} 4+\mathrm{O}$ allows for additional chain braching which further consumes the ethylene. DCB attained better agreement at the lower temperatures than WP primarily due to the usage of a higher vinyl decomposition rate. 
Minor differences were exhibited by both mechanisms with regards to the predicted methanol and ethane profiles. These predicted profiles did not compare well with the experimental data and indicates a problem symptomatic to both mechanisms. The problem either lies in a lack of methyl radical production (which leads to methanol and ethane formation) or inconsistencies within the methyl radical removal kinetic rates and/or kinetics.

\section{VINYL RADICAL KINETICS AND THERMOCHEMISTRY}

The vinyl radical intermediate plays an integral role in ethylene oxidation due to the importance of $\mathrm{C} 2 \mathrm{H} 4+\mathrm{OH}=\mathrm{C} 2 \mathrm{H} 3+\mathrm{H} 2 \mathrm{O}$. Unfortunately, the kinetics and product formation involving the vinyl destruction process is not well understood. Furthermore, the uncertainty of the vinyl radical heat of formation affects calculated thermodynamic equilibrium rates with considerable sensitivity. The important vinyl removal kinetics were assessed by comparing DCB and WP's choices to known evaluations and reviews. This would determine the relative importance each model placed on vinyl decomposition with respect to the ethylene oxidation modeling.

Table 1 represents a listing of typically assigned products and rate expressions involving the important vinyl oxidation and decomposition reactions used in the current literature and modeling studies. A wide range of values are exhibited for the $\mathrm{C} 2 \mathrm{H} 3+\mathrm{O} 2$ pathway to $\mathrm{C} 2 \mathrm{H} 2+\mathrm{HO} 2$ and vinyl decomposition rates. The former is due to the uncertainty in product domination for vinyl oxidation at typical combustion temperatures (i.e. $\mathrm{T}>900 \mathrm{~K}$ ) and the later is due to a poor understanding of the vinyl decomposition process.

The vinyl radical heat of formation has been measured or reviewed for a considerable range of values as exhibited in Table 1. The choice of 
Table 1

The Main Vinyl Oxidation and Decomposition Reaction Rate Expressions and Vinyl Heat of Formation as used by Modellers or cited in the Kinetics Literature Rate Data in $\mathrm{cm}^{3}$-mole-sec-kcal units

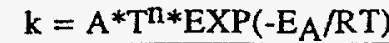

\begin{tabular}{|c|c|c|c|c|c|}
\hline Source & $\log A$ & $\mathbf{n}$ & $\mathrm{E}_{\mathrm{A}}$ & $\log k(1153 K)$ & Ref. \\
\hline \multicolumn{6}{|c|}{$\left.\left.\mathrm{C}_{2} \mathrm{H}_{3}+\mathrm{O}_{2}=\mathrm{CH} 2 \mathrm{O}+\mathrm{HCO}^{\mathrm{a}}\right) \mathrm{e}\right)$} \\
\hline Slagle et al. & 12.60 & 0.0 & -0.248 & 12.647 & 29 \\
\hline DC B & 12.48 & 0.0 & -0.25 & 12.527 & 3 \\
\hline WP & 12.176 & 0.0 & 0.0 & 12.176 & 2 \\
\hline Westmoreland $b)$ & 26.651 & -4.55 & 5.48 & 11.681 & 30 \\
\hline Bozzelli/Dean $b$ ) & 23.037 & -3.29 & 3.892 & 12.226 & 31 \\
\hline \multicolumn{6}{|c|}{$\left.\mathrm{C} 2 \mathrm{H} 3+\mathrm{O} 2=\mathrm{C} 2 \mathrm{H} 2+\mathrm{HO}_{2} \mathrm{e}\right)$} \\
\hline Tsang/Hampson & 11.08 & 0.0 & 0.0 & 11.08 & 4 \\
\hline Glarborg & 12.73 & 0.0 & 0.0 & 12.73 & 32 \\
\hline Warnatz & 12.0 & 0.0 & 0.0 & 12.0 & 5 \\
\hline \multicolumn{6}{|l|}{ Westmoreland $\left.{ }^{b}\right)$} \\
\hline Westmoreland & 11 & & 00 & & \\
\hline metathesis rxn. & 11.6 & 0.0 & 0.0 & 11.6 & 30 \\
\hline Bozzelli/Dean b) & 15.715 & -1.26 & 3.31 & 11.23 & 31 \\
\hline \multicolumn{6}{|c|}{ DCB and WP did not include this reaction pathway } \\
\hline \multicolumn{6}{|c|}{$\mathrm{C} 2 \mathrm{H} 3(+\mathrm{M})=\mathrm{C} 2 \mathrm{H} 2+\mathrm{H}(+\mathrm{M})$ a)c $) \mathrm{f})$} \\
\hline DCB & 44.323 & -8.447 & 51.106 & 8.77 & 3 \\
\hline WP & 28.58 & -4.4 & 44.29 & 6.71 & 2 \\
\hline Tsang/Hampson & 44.715 & -9.8 & 52.24 & 4.81 & 4 \\
\hline Baulch & 42.352 & -9.2 & 49.14 & 4.87 & 25 \\
\hline Warnatz & 13.567 & -1.0 & 32.06 & 4.43 & 5 \\
\hline Miller / Bowman & 25.639 & -4.4 & 42.52 & 4.11 & 26 \\
\hline KWKWd) & $36.79 \pm 0.4$ & -8.17 & 45.59 & 3.13 & 28 \\
\hline Rao/Skinnerg) & 13.73 & -1.3 & 33.97 & 3.31 & 27,5 \\
\hline Source & \multicolumn{2}{|c|}{$\Delta \mathrm{H}_{\mathrm{f}}(298 \mathrm{~K}, \mathrm{C} 2 \mathrm{H} 3)$} & Ref. & & \\
\hline \multirow{3}{*}{$\begin{array}{c}\text { Ayranci / Back } \\
\text { Shiromaru } \\
\text { Golden }\end{array}$} & 63.4 & $1 / \mathrm{mol}$ & 32 & & \\
\hline & \multicolumn{2}{|c|}{77} & 33 & & \\
\hline & \multicolumn{2}{|c|}{66} & 27 & & \\
\hline \multirow{2}{*}{\multicolumn{2}{|c|}{ McMillian / Golden }} & & 35 & & \\
\hline & & & 36 & & \\
\hline Kee et al. & \multicolumn{2}{|c|}{$\begin{array}{l}71.7 \\
68.4\end{array}$} & 21 & & \\
\hline
\end{tabular}

a) Corrected for fall-off at $1.0 \mathrm{~atm}$. b) Based on $\Delta \mathrm{H}_{\mathrm{f}}(\mathrm{C} 2 \mathrm{H} 3,298 \mathrm{~K})=70.4 \mathrm{kcal} / \mathrm{mol}$

c) Based on $\Delta \mathrm{H}_{\mathrm{f}}(\mathrm{C} 2 \mathrm{H} 3,298 \mathrm{~K})=68.4 \mathrm{kcal} / \mathrm{mol}$

d) KWKW performed RRKM calculations based on $\Delta \mathrm{H}_{\mathrm{f}}(\mathrm{C} 2 \mathrm{H} 3,298 \mathrm{~K})=63.4 \mathrm{kcal} / \mathrm{mol}$. This study has adjusted the activation energy downward from $50.59 \mathrm{kcal} / \mathrm{mol}$ to $45.59 \mathrm{kcal} / \mathrm{mol}$ to obtain a comparison to other rate expression evaluations performed at $\Delta \mathrm{H}_{\mathrm{f}}(\mathrm{C} 2 \mathrm{H} 3,298 \mathrm{~K})=68.4 \mathrm{kcal} / \mathrm{mol}$.

e) Rate expression units: $\mathrm{cm}^{3} / \mathrm{mol}-\mathrm{sec}$ f) Rate expression units: $\mathrm{sec}^{-1}$

g) Used $\mathrm{k}_{\infty}, \mathrm{C} 2 \mathrm{H} 2+\mathrm{H}=\mathrm{C} 2 \mathrm{H} 3$ from Warnatz [5] for Lindemann-Hinshelwood pressure correction. Equilibrium constants calculated from Kee et al. thermochemistry [21]. 
heat of formation value used may have an impact on the modeling calculations. Thermodynamic considerations aptly point out that the higher heat of formation value implies less stability of the vinyl radical which in turn favors higher rate expressions for vinyl decomposition or oxidation reactions and vice-versa. In this current study, no sensitivity analysis was conducted to assess which is the better heat of formation value.

\section{Vinyl Decomposition}

A compiled list of $\mathrm{C} 2 \mathrm{H} 3(+\mathrm{M})=\mathrm{C} 2 \mathrm{H} 2+\mathrm{H}(+\mathrm{M})$ rate expressions corrected for fall-off have been derived and are presented in Table 1. Curved Arrhenius plots of these expressions are presented in figure 3 . The list includes the reviews by Baulch et al. [25], Tsang and Hampson [4], and Warnatz [5] plus the recommendation from Miller and Bowman [26]. Recent shock tube studies of Rao and Skinner [27], and Kiefer, Wei, Kern and Wu (KWKW) [28] have been included in the assessment of the vinyl decomposition, reaction at high temperatures.

Pressure correction was performed according to the information presented by the various evaluations, and are based on a $68.4 \mathrm{kcal} / \mathrm{mol}$ heat of formation value. The referenced sources of these evaluations and applied pressure corrections have been conducted in the following manner. Tsang and Hampson obtained a RRKM calculated low pressure rate expression based on the forward rate expression determined by Benson and Haugen [37] plus the measured low temperature and high pressure reverse rates of Payne and Stief [38] and Sugarawa et al. [39]. The Tsang and Hampson low pressure rate expression was corrected for weak collisions by using the recommended collisional deactivation step size of $450 \mathrm{~cm}^{-1}$ for a $N_{2}$ collider at one atmosphere. Table 1 lists the Tsang and Hampson pressure corrected rate evaluation. Baulch et al. [25] recommended the use of the Tsang and Hampson vinyl decomposition low pressure rate expression but advised the use of the Troe Method for fall-off corrections. The high pressure forward rate 


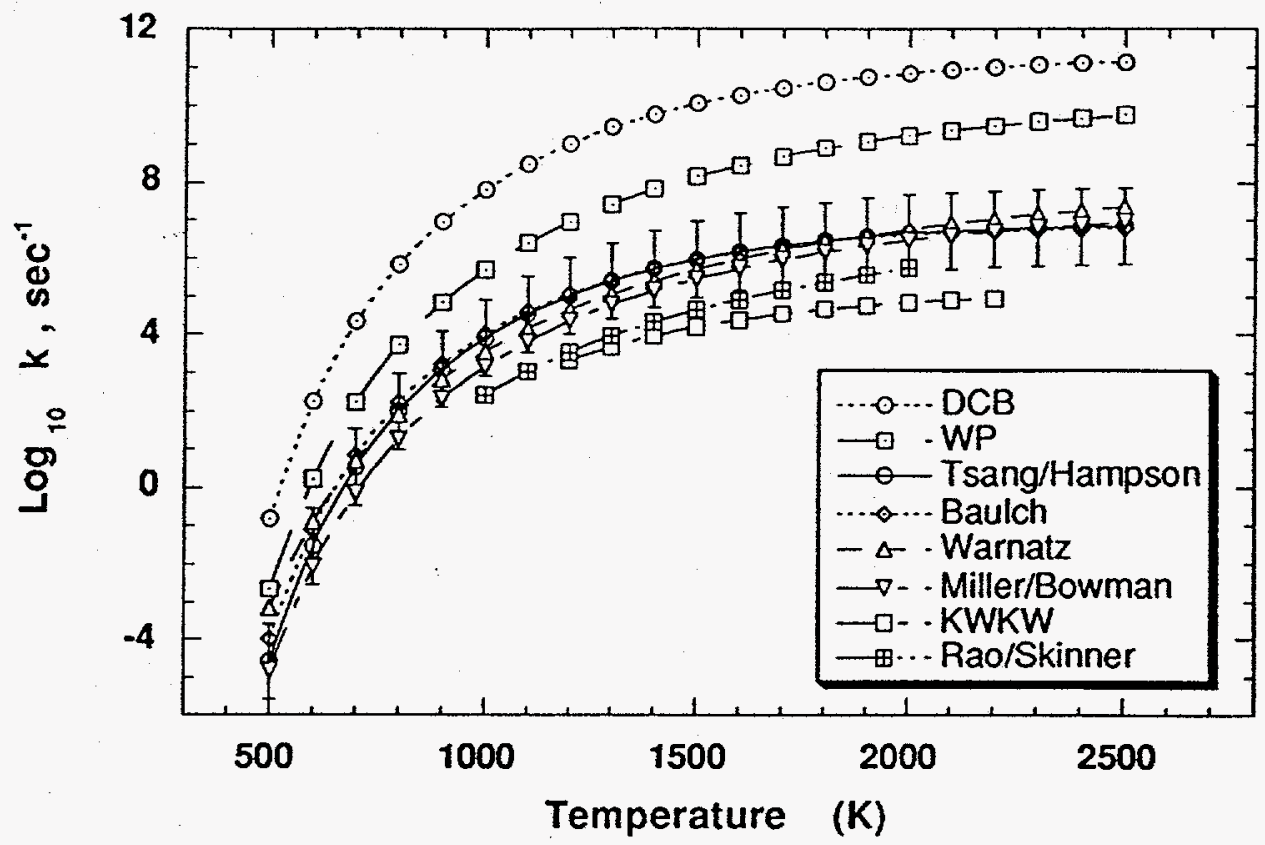

Figure 3: Evaluated kinetic rate data at one atmosphere for vinyl decomposition based on $\Delta \mathrm{H}_{\mathrm{f}}(\mathrm{C} 2 \mathrm{H} 3,298 \mathrm{~K})=68.4 \mathrm{kcal} / \mathrm{mol}$. See Table 1 for the numerical representation of the fall-off corrected kinetic rates.

expression was taken from the Warnatz review and a center-broadening parameter value of 0.35 as recommended by Baulch et al. was then used to derive the Table 1 expression. The Warnatz [5] recommended low and high pressure forward rate expressions were based on literature data taken from the 1960's and 1970's. The Lindemann-Hinshelwood method was then applied to derive the rate expression ascribed to Warnatz in Table 1. All reviews have an associated uncertainty factor of 10 and, along with the Miller and Bowman recommendation, lie within this measure of uncertainty. This is noted by the uncertainty factor bars placed about the Tsang and Hampson rate expression shown in the figure. Clearly, all reviews are in accordance with one another. 
A comparison of the reviewed vinyl decomposition kinetics with recent shock tube measurements indicates slower vinyl decomposition kinetics occurs than suggested by the recent reviews. The high temperature vinyl-bromide and vinyl-iodide pyrolysis shock tube measurements of $\mathrm{Rao}$ and Skinner indicate the vinyl decomposition rate occurs a factor of 10 lower than the recent reviews. Their vinyl decomposition results were determined independently of the vinyl radical heat of formation and support the revised rate expression of KWKW as determined by this study. The high temperature 1,3-butadiene pyrolysis shock tube measurements and RRKM analysis of KWKW suggest a relatively stable vinyl radical and a slow vinyl decomposition kinetic rate in direct contradiction to WP and $\mathrm{DCB}$.

The WP and DCB mechanisms utilize vinyl decomposition kinetic rates which exceed the upper uncertainty factor limit for the reviewed evaluations by approximately one and three orders of magnitude.

Furthermore, $W P$ and $D C B$ usage of fast vinyl decomposition rates are not supported by the Rao/Skinner and KWKW shock tube studies. The analysis of the vinyl decomposition process coupled with the poor acetylene modeling predictions of DCB and WP clearly suggest that a lower vinyl decomposition kinetic rate must be used than those supported by $\mathrm{DCB}$ and WP.

Vinyl Oxidation by Molecular Oxygen

The dearth of high temperature studies concerning the reaction kinetics of $\mathrm{C} 2 \mathrm{H} 3+\mathrm{O} 2$ is the primary cause of uncertainty in product formation and kinetic rates at typical combustion conditions. However, the kinetics concerning $\mathrm{C} 2 \mathrm{H} 3+\mathrm{O} 2$ is well-defined at low temperatures. Experiments by Baldwin and Walker [40] and Gutman et al. [29, 41] indicated that the addition channel leading to $\mathrm{CH} 2 \mathrm{O}+\mathrm{HCO}$ is the primary products for $\mathrm{C} 2 \mathrm{H} 3+\mathrm{O} 2$ at low temperatures. Gutman et al. measured rate constants and conclusively established that $\mathrm{C} 2 \mathrm{H} 3+\mathrm{O} 2$ formed $\mathrm{CH} 2 \mathrm{O}+\mathrm{HCO}$ 
at 297-599K and 0.48-3.6 torr He. No $\mathrm{C} 2 \mathrm{H} 2$ and $\mathrm{HO} 2$ were detected, so any $\mathrm{C} 2 \mathrm{H} 2+\mathrm{HO} 2$ product pathway contribution is considered to be less than $10 \%$.

No direct high temperature chemical kinetic studies have been conducted to examine the $\mathrm{C} 2 \mathrm{H} 3+\mathrm{O} 2$ reaction. However, Cooke and Williams [42] studied ignition delays in shock-heated ethane-oxygenargon mixtures at $1400-1800 \mathrm{~K}$ and $200-300$ torr. Their measured induction times were modelled with a 34-step mechanism that included the $\mathrm{C} 2 \mathrm{H} 3+\mathrm{O} 2=\mathrm{C} 2 \mathrm{H} 2+\mathrm{HO} 2$ reaction.

Reviews by Tsang and Hampson [4] and Warnatz [5] assumed the $\mathrm{C} 2 \mathrm{H} 3$ $+\mathrm{O} 2$ reaction proceeded to $\mathrm{C} 2 \mathrm{H} 2+\mathrm{HO}_{2}$ in an analogous fashion as the $\mathrm{C} 2 \mathrm{H} 5+\mathrm{O} 2$ reaction which was thought to proceed by simple $\mathrm{H}$-atom abstraction by $\mathrm{O} 2$ under typical combustion conditions. However, the recent study by Wagner et al. [43] postulated that the ethyl radical plus oxygen reaction is complex. The reaction involves $\mathrm{O} 2$ addition followed by stabilization of the adduct (only for temperatures below approximately $650 \mathrm{~K}$ ) or isomerization of the adduct followed by decomposition to $\mathrm{C} 2 \mathrm{H} 4+\mathrm{HO} 2$. The postulated reaction scheme effectively rules out the direct $\mathrm{H}$-atom metathesis route for $\mathrm{C} 2 \mathrm{H} 5+\mathrm{O} 2$ and therefore suggests that $\mathrm{H}$-atom abstraction from $\mathrm{C} 2 \mathrm{H} 3$ by $\mathrm{O} 2$ is unlikely to occur. Unfortunately, the viability of the simple $\mathrm{H}$-atom abstraction by $\mathrm{O} 2$ reaction mechanism is not known for the $\mathrm{C} 2 \mathrm{H} 3+\mathrm{O} 2$ reaction. Interestingly, Westmoreland [30] suggested the possibility that $\mathrm{C} 2 \mathrm{H} 3+$ $\mathrm{O} 2$ metathesis reaction leading to $\mathrm{C} 2 \mathrm{H} 2+\mathrm{HO} 2$ could dominate at temperatures above $1500 \mathrm{~K}$ depending on the prescribed nature of the fall-off for the $\mathrm{C} 2 \mathrm{H} 3+\mathrm{O} 2=\mathrm{CH} 2 \mathrm{O}+\mathrm{HCO}$ reaction.

If the primary oxidation channel is $\mathrm{CH} 2 \mathrm{O}+\mathrm{HCO}$ at high temperatures, then the early source of reactive free radicals must come from formyl radical dissociation. The $\mathrm{H}$-atom produced from formyl decomposition would be sufficient to sustain chain branching and further ethylene oxidation. The other possibility, $\mathrm{C} 2 \mathrm{H} 2+\mathrm{HO} 2$, is ruled out due to poor 
predictions of the acetylene profile that would result if this reaction were to be favored and the production of an unreactive $\mathrm{HO} 2$ radical.

Lastly, vinyl plus a radical reaction is unlikely to provide the necessary impetus for substantial reactive free radical production as the $\mathrm{C} 2 \mathrm{H} 3+\mathrm{O} 2$ reaction dominates at these temperatures.

\section{THIS STUDY'S ETHYLENE OXIDATION MODEL}

The inadequacies represented by the poorly predicted acetylene, methanol and other reaction intermediate profiles, and the disagreement at the higher temperatures of the data set by the WP and DCB mechanisms warranted the development of this studies ethylene oxidation model. A review of kinetics literature was conducted for the development and compiliation of this mechanism [23].

The presently developed model was used to reproduce the ethylene oxidation experimental measurements. of Thornton and the lean, atmospheric pressure, methane and ethane data sets for the $900-1200 \mathrm{~K}$ temperature range of Dagaut et al. $[3,44]$. Discussion will pertain only to the ethylene oxidation chemistry, further discussion on the methane and ethane modeling is presented in Marinov [23]. The ethylene mechanism is listed in Table 2 and consists of 206 reactions and 40 species. The inclusion of third body efficiencies and pressure corrections were applied as necessary to the model. Table 3 shows the species thermochemistry used in this study.

\section{Discussion}

\section{Ethylene Kinetics:}

For the temperature range 1053 to $1253 \mathrm{~K}$ of this study, the major loss of ethylene occurs by reaction (50) of Table 2:

$$
\mathrm{C} 2 \mathrm{H} 4+\mathrm{OH}=\underset{13}{\mathrm{C} 2 \mathrm{H} 3}+\mathrm{H} 2 \mathrm{O}
$$


Table 2

Ethylene oxidation mechanism. Rate data in $\mathrm{cm}^{3}$-mole-sec-kcal units, $k=A^{*} T^{n *} \exp \left(-E_{A} / R T\right)$

\begin{tabular}{|c|c|c|c|c|c|c|c|c|c|c|c|}
\hline No. & \multicolumn{7}{|c|}{ Reaction } & $\log A$ & $\mathbf{n}$ & $E_{A}$ & Ref. \\
\hline $\begin{array}{l}1 . \\
2 . \\
3 . \\
4 . \\
5 . \\
6 . \\
7 . \\
8 .\end{array}$ & $\begin{array}{l}H \\
H \\
H \\
H \\
O \\
O H \\
O \\
H\end{array}$ & $\begin{array}{l}H \\
H \\
H \\
H \\
H \\
H \\
O \\
O 2\end{array}$ & $\begin{array}{c}M \\
H 2 \\
H 2 O \\
C O 2 \\
M^{*} \\
M^{*} \\
M^{*} \\
M^{\#}\end{array}$ & $\begin{array}{l}= \\
= \\
= \\
= \\
= \\
= \\
= \\
=\end{array}$ & $\begin{array}{l}\mathrm{H2} \\
\mathrm{H} 2 \\
\mathrm{H} 2 \\
\mathrm{H} 2 \\
\mathrm{OH} \\
\mathrm{H} 2 \mathrm{O} \\
\mathrm{O} 2 \\
\mathrm{HO} 2\end{array}$ & $\begin{array}{l}\mathrm{H} 2 \\
\mathrm{H} 2 \mathrm{O} \\
\mathrm{CO} 2\end{array}$ & $\begin{array}{l}\mathbf{M}^{*} \\
\mathbf{M}^{*} \\
\mathbf{M}^{*} \\
\mathbf{M}^{\#}\end{array}$ & $\begin{array}{c}18.0 \\
16.964 \\
19.778 \\
20.74 \\
18.673 \\
22.345 \\
13.275 \\
17.903\end{array}$ & $\begin{array}{c}-1.0 \\
-0.6 \\
-1.25 \\
-2.0 \\
-1.0 \\
-2.0 \\
0.0 \\
-0.8\end{array}$ & $\begin{array}{l}0.0 \\
0.0 \\
0.0 \\
0.0 \\
0.0 \\
0.0 \\
-1.788 \\
0.0\end{array}$ & $\begin{array}{l}{[45]} \\
{[45]} \\
{[45]} \\
{[45]} \\
{[4]} \\
{[4]} \\
{[4]} \\
{[25]}\end{array}$ \\
\hline 9. & $\mathrm{OH}$ & $\mathrm{OH}$ & $(M)$ & $=$ & $\mathrm{H} 2 \mathrm{O} 2$ & & $(M) \dagger$ & 14.092 & -0.37 & 0.0 & {$[46,4, a]$} \\
\hline $\begin{array}{l}10 . \\
11 .\end{array}$ & $\begin{array}{c}\mathrm{H} 2 \mathrm{O} \\
\mathrm{H} 2\end{array}$ & $\begin{array}{c}O \\
O H\end{array}$ & & $\begin{array}{l}= \\
=\end{array}$ & $\begin{array}{c}\mathrm{OH} \\
\mathrm{H} 2 \mathrm{O}\end{array}$ & $\underset{\mathrm{H}}{\mathrm{OH}}$ & & $\begin{array}{c}5.171 \\
8.0\end{array}$ & $\begin{array}{l}2.6 \\
1.6\end{array}$ & $\begin{array}{l}15.175 \\
3.298\end{array}$ & $\begin{array}{l}{[47]} \\
{[25]}\end{array}$ \\
\hline 12. & $\mathrm{O} 2$ & $\mathrm{H}$ & & $=$ & $\mathrm{OH}$ & 0 & & 16.546 & -0.7 & 17.07 & [48] \\
\hline 13. & $H$ & $\mathrm{HO} 2$ & & $=$ & He & $\mathrm{O} 2$ & & 13.63 & 0.0 & 1.41 & [25] \\
\hline 14. & $\mathrm{H}$ & $\mathrm{HO2}$ & & $=$ & $\mathrm{OH}$ & $\mathrm{OH}$ & & 14.22 & 0.0 & 0.87 & [25] \\
\hline 15. & $H$ & 102 & & $=$ & $\mathrm{H} 2 \mathrm{O}$ & 0 & & 13.48 & 0.0 & 1.72 & [25] \\
\hline 16. & $\begin{array}{c}\mathrm{O} \\
\mathrm{OH}\end{array}$ & HO2 & & $=$ & $\mathrm{OH}$ & $\mathrm{O} 2$ & & 13.26 & 0.0 & -0.397 & [4] \\
\hline $\begin{array}{l}17 . \\
18\end{array}$ & $\begin{array}{c}\mathrm{OH} \\
\mathrm{H} 2 \mathrm{O} 2\end{array}$ & $\begin{array}{c}\mathrm{HO} 2 \\
\mathrm{OH}\end{array}$ & & $=$ & $\begin{array}{l}\mathrm{H}_{2} \mathrm{O} \\
\mathrm{HO} 2\end{array}$ & $\begin{array}{c}\mathrm{O} 2 \\
\mathrm{H} 2 \mathrm{O}\end{array}$ & & 13.46 & 0.0 & -0.497 & [25] \\
\hline 18. & $\begin{array}{l}\mathrm{H} 2 \mathrm{O} 2 \\
\mathrm{H} 2 \mathrm{O} 2\end{array}$ & $\begin{array}{c}\mathrm{OH} \\
\mathrm{O}\end{array}$ & & $=$ & $\begin{array}{l}\mathrm{HO} 2 \\
\mathrm{OH}\end{array}$ & $\begin{array}{l}\mathrm{H} 2 \mathrm{O} \\
\mathrm{HO} 2\end{array}$ & & 0.381 & 4.042 & -2.126 & (b) \\
\hline $\begin{array}{l}19 . \\
20 .\end{array}$ & $\begin{array}{l}\mathrm{H} 2 \mathrm{O} 2 \\
\mathrm{H} 2 \mathrm{O} 2\end{array}$ & $\begin{array}{l}\mathrm{O} \\
\mathrm{H}\end{array}$ & & $\begin{array}{l}= \\
=\end{array}$ & $\begin{array}{l}\mathrm{OH} \\
\mathrm{HO} 2\end{array}$ & $\begin{array}{c}\mathrm{HO}_{2} \\
\mathrm{H} 2\end{array}$ & & 6.984 & 2.0 & 3.974 & [4] \\
\hline 21. & $\mathrm{H} 2 \mathrm{O} 2$ & $\mathrm{H}$ & & $\begin{array}{l}= \\
=\end{array}$ & $\mathrm{H}_{2} \mathrm{O}$ & $\mathrm{OH}$ & & $\begin{array}{c}12.23 \\
13.382\end{array}$ & $\begin{array}{l}0.0 \\
0.0\end{array}$ & $\begin{array}{l}3.775 \\
3.974\end{array}$ & $\begin{array}{l}{[25]} \\
{[4]}\end{array}$ \\
\hline 22. & $\mathrm{HO} 2$ & $\mathrm{HO} 2$ & & $=$ & $\mathrm{H} 2 \mathrm{O} 2$ & $\mathrm{O} 2$ & & -14.06 & 8.1 & -8.68 & {$[49]$} \\
\hline 23. & $\mathrm{H} 2$ & 02 & & $=$ & $\mathrm{OH}$ & $\mathrm{OH}$ & & 13.23 & 0.0 & 47.78 & [25] \\
\hline 24. & 0 & $\mathrm{H} 2$ & & $=$ & OHs & $H$ & & 4.705 & 2.67 & 6.29 & [50] \\
\hline 25. & $\infty$ & $\mathrm{OH}$ & & $=$ & $\mathrm{CO} 2$ & $H$ & & 6.643 & 1.5 & -0.741 & {$[5]$} \\
\hline 26. & $\mathrm{CO}$ & 0 & $M^{*}$ & $=$ & $\mathrm{CO} 2$ & & $M^{*}$ & 14.79 & 0.0 & 3.00 & [4] \\
\hline 27. & co & $\mathrm{HO} 2$ & & $=$ & $\mathrm{CO} 2$ & $\mathrm{OH}$ & & 14.18 & 0.0 & 23.64 & [5] \\
\hline 28. & $\infty$ & $\mathrm{O} 2$ & & $=$ & $\mathrm{CO} 2$ & 0 & & 12.4 & 0.0 & 47.7 & [5] \\
\hline 29. & $\mathrm{CH} 3$ & $\mathrm{CH} 3$ & (M) & $=$ & $\mathrm{C} 2 \mathrm{H} 6$ & & (M) & 16.964 & -1.174 & 0.636 & {$[51, a]$} \\
\hline 30. & $\mathrm{C} 2116$ & $\mathrm{OH}$ & & $=$ & $\mathrm{C} 2 \mathrm{H} 5$ & $\mathrm{H} 2 \mathrm{O}$ & & 6.859 & 2.0 & 0.864 & [25] \\
\hline 31. & $\mathrm{C} 2 \mathrm{H} 6$ & 0 & & $=$ & $\mathrm{C} 2 \mathrm{H5}$ & $\mathrm{OH}$ & & 10.941 & 0.957 & 6.637 & [52] \\
\hline 32. & C2+t6 & $H$ & & $=$ & $\mathrm{C} 24 \mathrm{H}$ & $\mathrm{H} 2$ & & 2.73 & 3.5 & 5.21 & [4] \\
\hline 33. & $\mathrm{C} 2 \mathrm{H} 16$ & $\mathrm{HO} 2$ & & $=$. & $\mathrm{C} 2 \mathrm{HS}$ & $\mathrm{H}_{2} \mathrm{O} 2$ & & 11.47 & 0.0 & 14.94 & [4] \\
\hline 34. & $\mathrm{C} 2 \mathrm{H} 6$ & $\mathrm{CH} 3$ & & $=$ & $\mathrm{C2H} 5$ & $\mathrm{CH} 4$ & & -6.822 & 6.0 & 6.046 & [25] \\
\hline 35. & $\mathrm{C} 2 \mathrm{H} 6$ & & (M) & $=$ & $\mathrm{C2H}$ & $H$ & $(M)$ & 20.947 & -1.22 & 102.2 & {$[53, c]$} \\
\hline 36. & $\mathrm{C} 2 \mathrm{H} 6$ & $\mathrm{O} 2$ & & $=$ & $\mathrm{C} 2 \mathrm{HS}$ & $\mathrm{HO} 2$ & & 13.6 & 0.0 & 50.87 & [4] \\
\hline 37. & $\mathrm{C} 2 \mathrm{H} 5$ & $\mathrm{OH}$ & & $=$ & $\mathrm{C} 2 \mathrm{H}+4$ & $\mathrm{H} 2 \mathrm{O}$ & & 13.38 & 0.0 & 0.0 & [4] \\
\hline 38. & $\mathrm{C} 2 \mathrm{H} 5$ & $\mathrm{OH}$ & & $=$ & $\mathrm{CH} 3$ & $\mathrm{CH} 2 \mathrm{O}$ & $H$ & 13.38 & 0.0 & 0.0 & [4] \\
\hline 39. & $\mathrm{C} 2 \mathrm{H} 5$ & 0 & & $=$ & $\mathrm{CH}_{3}$ & $\mathrm{CH} 2 \mathrm{O}$ & & 13.645 & 0.0 & 0.0 & [54] \\
\hline 40. & $\mathrm{C2H5}$ & $\mathrm{O}$ & & $=$ & $\mathrm{CH} B \mathrm{CHO}$ & $H$ & & 13.727 & 0.0 & 0.0 & [54] \\
\hline 41. & $\mathrm{C} 2 \mathrm{H} 5$ & 0 & & $=$ & $\mathrm{CaH} 4$ & $\mathrm{OH}$ & & 13.512 & 0.0 & 0.0 & {$[54]$} \\
\hline 42. & $\mathrm{C} 2 \mathrm{H} 5$ & $\mathrm{HO} 2$ & & $=$ & $\mathrm{CH} 3$ & $\mathrm{CH} 2 \mathrm{O}$ & $\mathrm{OH}$ & 13.2 & 0.0 & 0.0 & {$[4, d]$} \\
\hline 43. & $\mathrm{C} 2 \mathrm{H} 5$ & $\mathrm{C} 2 \mathrm{H} 5$ & & $=$ & $\mathrm{C} 2 \mathrm{H}_{4}$ & $\mathrm{C} 2 \mathrm{H} 6$ & & 12.14 & 0.0 & 0.0 & {$[4]$} \\
\hline 44. & $\mathrm{C} 2 \mathrm{HS}$ & $\mathrm{CH} 3$ & & $=$ & $\mathrm{C} 2 \mathrm{H}_{4}$ & $\mathrm{CH}_{4}$ & & -3.36 & 5.0 & 8.3 & [3] \\
\hline 45. & $\mathrm{C} 2 \mathrm{H} 5$ & $H$ & & $=$ & $\mathrm{C}_{2} \mathrm{H}_{4}$ & $\mathrm{H}_{2}$ & & 14.1 & 0.0 & 8.0 & [3] \\
\hline 46. & $\mathrm{C} 2 \mathrm{H} 5$ & HCO & & $=$ & $\mathrm{C} 2 \mathrm{H} 6$ & $\mathrm{CO}$ & & 14.0 & 0.0 & 0.0 & [4] \\
\hline 47. & $\mathrm{C} 2 \mathrm{H} 5$ & $\mathrm{O} 2$ & & $=$ & $\mathrm{C} 2 \mathrm{H}_{4}$ & $\mathrm{HO} 2$ & & 20.48 & -2.86 & 6.76 & [55] \\
\hline 48. & $\mathrm{C} 2 \mathrm{H} 5$ & $\mathrm{O} 2$ & & $=$ & $\mathrm{C} 2 \mathrm{H} 44 \mathrm{O}_{2} \mathrm{H}$ & & & 40.11 & -9.41 & 11.03 & [55] \\
\hline 49. & $\mathrm{C} 2 \mathrm{HS}$ & $\mathrm{O} 2$ & & $=$ & $\mathrm{CH} 3 \mathrm{CHO}$ & $\mathrm{OH}$ & & 14.20 & -1.17 & 10.39 & [55] \\
\hline 50. & $\mathrm{C} 2 \mathrm{H4}$ & $\mathrm{OH}$ & & $=$ & $\mathrm{C} 2 \mathrm{H}_{3}$ & $\mathrm{H} 2 \mathrm{O}$ & & 6.47 & 2.0 & 2.235 & [56] \\
\hline 51. & $\mathrm{C} 2 \mathrm{H}_{4}$ & 0 & & $=$ & $\mathrm{CH} 3$ & $\mathrm{HCO}$ & & 13.74 & 0.0 & 5.5 & {$[57, e]$} \\
\hline 52. & $\mathrm{C} 2 \mathrm{H}_{4}$ & 0 & & $=$ & $\mathrm{CH} 2$ & $\mathrm{HCO}$ & $\mathrm{H}$ & 13.37 & 0.0 & 5.5 & {$[57, e]$} \\
\hline 53. & $\mathrm{C} 2 \mathrm{H} 4$ & $H$ & & $=$ & $\mathrm{C} 2 \mathrm{H} 3$ & $\mathrm{H}_{2}$ & & 7.18 & 2.0 & 6.0 & {$[9]$} \\
\hline 54. & $\mathrm{C} 2 \mathrm{H} 4$ & $\mathrm{CH} 3$ & & $=$ & $\mathrm{C}_{2} \mathrm{H}_{3}$ & $\mathrm{CH}_{4}$ & & 0.821 & 3.7 & 9.5 & [4] \\
\hline 55. & $\mathrm{C} 2 \mathrm{H}_{4}$ & $\mathrm{HO} 2$ & & $=$ & $\mathrm{C}_{2} \mathrm{H}_{3}$ & $\mathrm{H}_{2} \mathrm{O}_{2}$ & & 13.05 & 0.0 & 30.43 & {$[12]$} \\
\hline 56. & $\mathrm{C} 2 \mathrm{H} 4$ & $\mathrm{HO} 2$ & & $=$ & $\mathrm{C} 2 \mathrm{H} 4 \mathrm{O} 2 \mathrm{H}$ & & & 11.3 & 0.0 & 8.0 & [12] \\
\hline 57. & $\mathrm{C} 2 \mathrm{H}_{4}$ & $H$ & (M) & $=$ & $\mathrm{C} 2 \mathrm{H} 5$ & & $(\mathrm{M})$ & 13.42 & 0.0 & 2.16 & {$[58, a]$} \\
\hline 58. & $\mathrm{C} 2 \mathrm{H}_{4}$ & $\mathrm{O} 2$ & & $=$ & $\mathrm{C} 2 \mathrm{H} 3$ & $\mathrm{HO}_{2}$ & & 13.63 & 0.0 & 57.6 & [4] \\
\hline 59. & $\mathrm{C} 2 \mathrm{H} 4$ & & $(M)$ & $=$ & $\mathrm{C} 2 \mathrm{H}_{2}$ & $\mathrm{H} 2$ & (M) & 12.90 & 0.44 & 88.76 & {$[4, i]$} \\
\hline 60. & $\mathrm{C}_{2} \mathrm{H}_{4}$ & & $(M)$ & $=$ & $\mathrm{C} 2 \mathrm{H} 3$ & $H$ & (M) & 12.90 & 0.44 & 88.76 & {$[4,1]$} \\
\hline 61. & $\mathrm{C} 2 \mathrm{H} 4 \mathrm{O} 2 \mathrm{H}$ & & & $=$ & $\mathrm{C} 2 \mathrm{H} 4 \mathrm{O}$ & $\mathrm{OH}$ & & 11.5 & 0.0 & 19.5 & [12] \\
\hline 62. & $\mathrm{C} 2 \mathrm{H} 4 \mathrm{O}$ & $\mathrm{OH}$ & & $=$ & $\mathrm{C} 2 \mathrm{H} 3 \mathrm{O}$ & $\mathrm{H} 2 \mathrm{O}$ & & 13.68 & 0.0 & 5.955 & [12] \\
\hline 63. & $\mathrm{C} 2 \mathrm{H} 3 \mathrm{O}$ & & & $=$ & $\mathrm{CH} 3 \mathrm{CO}$ & & & 14.93 & 0.0 & 14.0 & [12] \\
\hline 64. & $\mathrm{CH} 2 \mathrm{CHO}$ & & & $=$ & $\mathrm{CH} 2 \mathrm{CO}$ & $H$ & & 37.02 & -7.189 & 44.32 & [g] \\
\hline 65. & $\mathrm{CH} 2 \mathrm{CH}$ & $\mathrm{O} 2$ & & $=$ & $\mathrm{CH}+2 \mathrm{CO}$ & $\mathrm{HO} 2$ & & 10.2 & 0.0 & 0.0 & [k98] \\
\hline $\begin{array}{l}66 . \\
67\end{array}$ & $\begin{array}{l}\mathrm{CHZCHO} \\
\mathrm{CH} C \mathrm{CHO}\end{array}$ & $\begin{array}{l}\mathrm{O} 2 \\
\mathrm{O} 2\end{array}$ & & $\begin{array}{l}= \\
=\end{array}$ & $\begin{array}{c}\mathrm{CH} 2 \mathrm{O} \\
\mathrm{CH}+\mathrm{CHO}\end{array}$ & $\mathrm{CO}$ & $\mathrm{OH}$ & $\begin{array}{l}10.4 \\
11.4\end{array}$ & $\begin{array}{l}0.0 \\
0.0\end{array}$ & 0.0 & {$[25]$} \\
\hline 68. & $\mathrm{CH} 2 \mathrm{CHO}$ & 0 & & $=$ & $\mathrm{CH}_{2} \mathrm{O}$ & HCO & & 13.6 & 0.0 & $\begin{array}{c}14.64 \\
0.0\end{array}$ & $\begin{array}{c}{\left[k_{160}\right]} \\
\text { (h) }\end{array}$ \\
\hline 69. & $\mathrm{CH} 2 \mathrm{CHOO}$ & $\mathrm{HO} 2$ & & $=$ & $\mathrm{CH} 2 \mathrm{O}$ & $\mathrm{HCO}$ & $\mathrm{OH}$ & 13.0 & 0.0 & 0.0 & $\left(k_{157}\right)$ \\
\hline
\end{tabular}




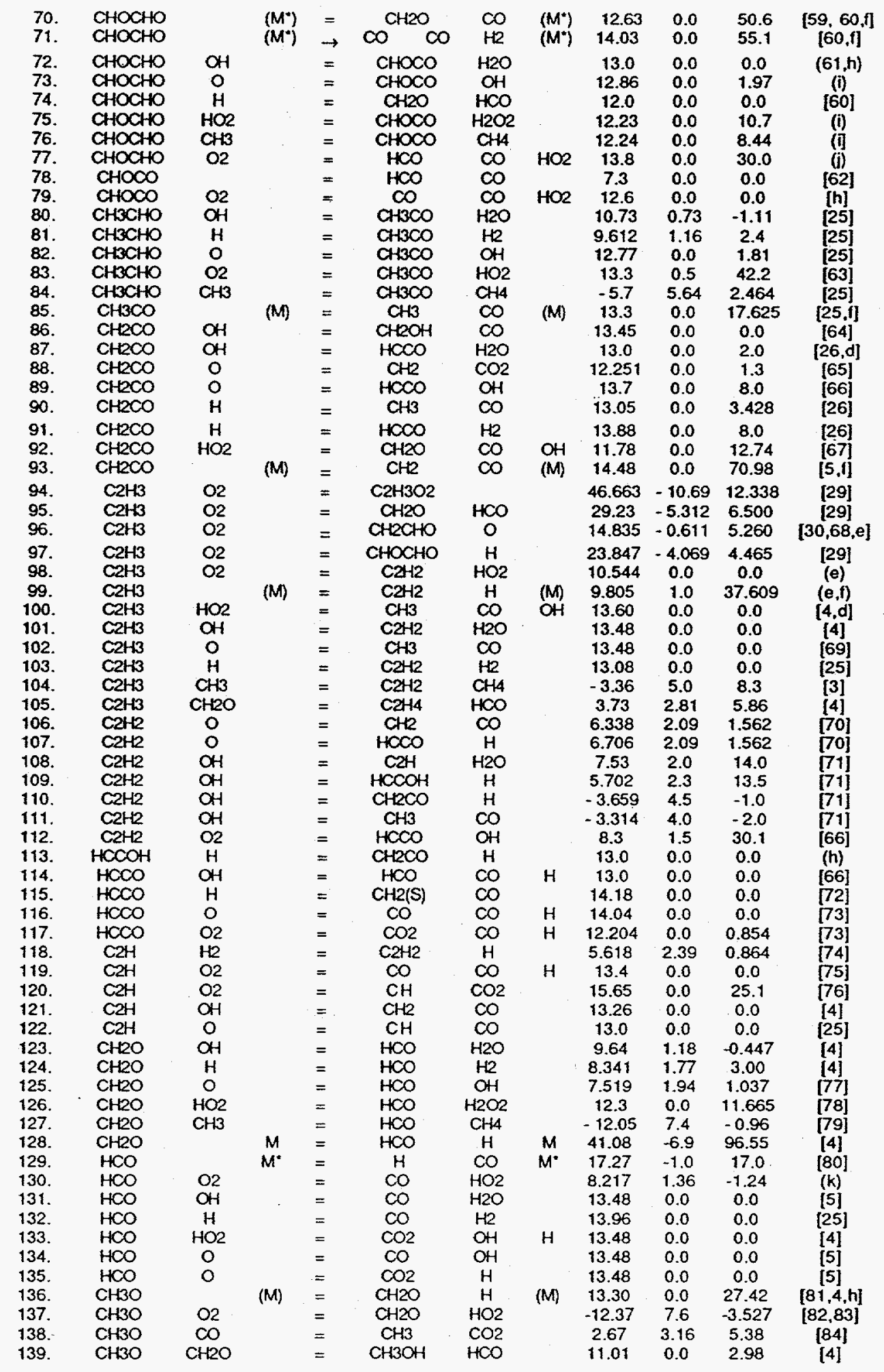




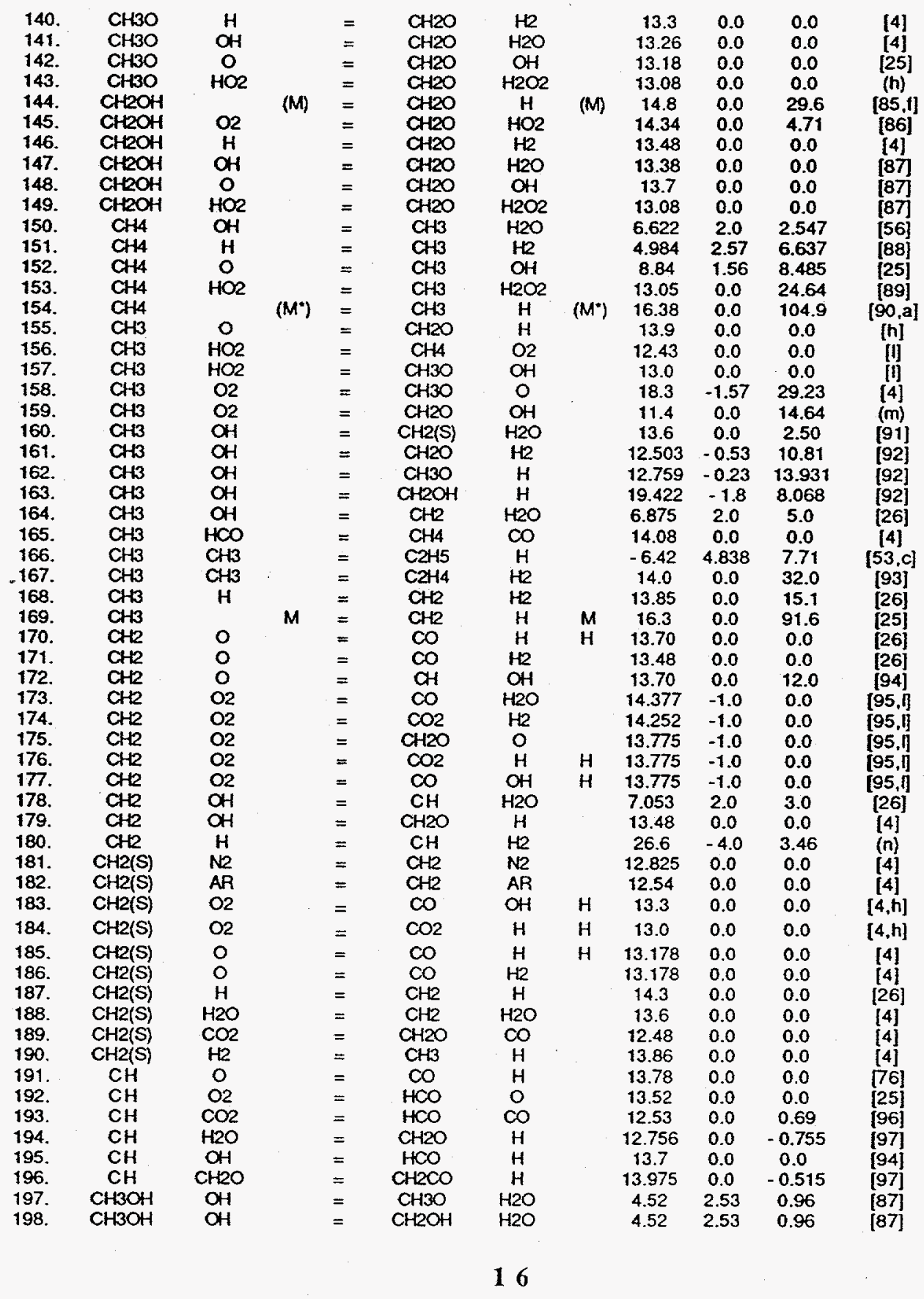




\begin{tabular}{|c|c|c|c|c|c|c|c|c|c|c|c|}
\hline $\begin{array}{l}199 . \\
200 . \\
201 . \\
202 . \\
203 . \\
204 . \\
205 . \\
206 .\end{array}$ & $\begin{array}{l}\mathrm{CH} 3 \mathrm{OH} \\
\mathrm{CH} 3 \mathrm{OH} \\
\mathrm{CH} 3 \mathrm{OH} \\
\mathrm{CH} 3 \mathrm{OH} \\
\mathrm{CH} 3 \mathrm{OH} \\
\mathrm{CH} 3 \mathrm{OH} \\
\mathrm{CH} 3 \mathrm{OH} \\
\mathrm{CH} 3 \mathrm{OH}\end{array}$ & $\begin{array}{c}\mathrm{H} \\
\mathrm{H} \\
\mathrm{O} \\
\mathrm{CH} \\
\mathrm{CH} \\
\mathrm{HO2} \\
\mathrm{O} 2\end{array}$ & $(\mathrm{M})$ & $\begin{array}{l}= \\
= \\
= \\
= \\
= \\
= \\
= \\
=\end{array}$ & $\begin{array}{l}\mathrm{CH} 2 \mathrm{OH} \\
\mathrm{CH} 3 \mathrm{O} \\
\mathrm{CH} 2 \mathrm{OH} \\
\mathrm{CH} 2 \mathrm{OH} \\
\mathrm{CH} 30 \\
\mathrm{CH} \\
\mathrm{CH} \mathrm{OH} \\
\mathrm{CH} \\
\mathrm{CH} 2 \mathrm{OH}\end{array}$ & $\begin{array}{c}\mathrm{H} 2 \\
\mathrm{H} 2 \\
\mathrm{OH} \\
\mathrm{CH} 4 \\
\mathrm{CH} \\
\mathrm{H} 2 \mathrm{O} 2 \\
\mathrm{OH} \\
\mathrm{HO} 2\end{array}$ & (M) & $\begin{array}{c}8.505 \\
6.623 \\
5.589 \\
1.5 \\
1.16 \\
13.6 \\
16.3 \\
13.31\end{array}$ & $\begin{array}{l}1.49 \\
2.11 \\
2.5 \\
3.17 \\
3.1 \\
0.0 \\
0.0 \\
0.0\end{array}$ & $\begin{array}{c}4.25 \\
4.868 \\
3.08 \\
7.17 \\
6.93 \\
19.4 \\
89.87 \\
44.91\end{array}$ & $\begin{array}{c}{[98]} \\
{[87]} \\
{[87]} \\
{[87]} \\
{[87]} \\
{[99]} \\
{[100,1]} \\
{[87]}\end{array}$ \\
\hline
\end{tabular}

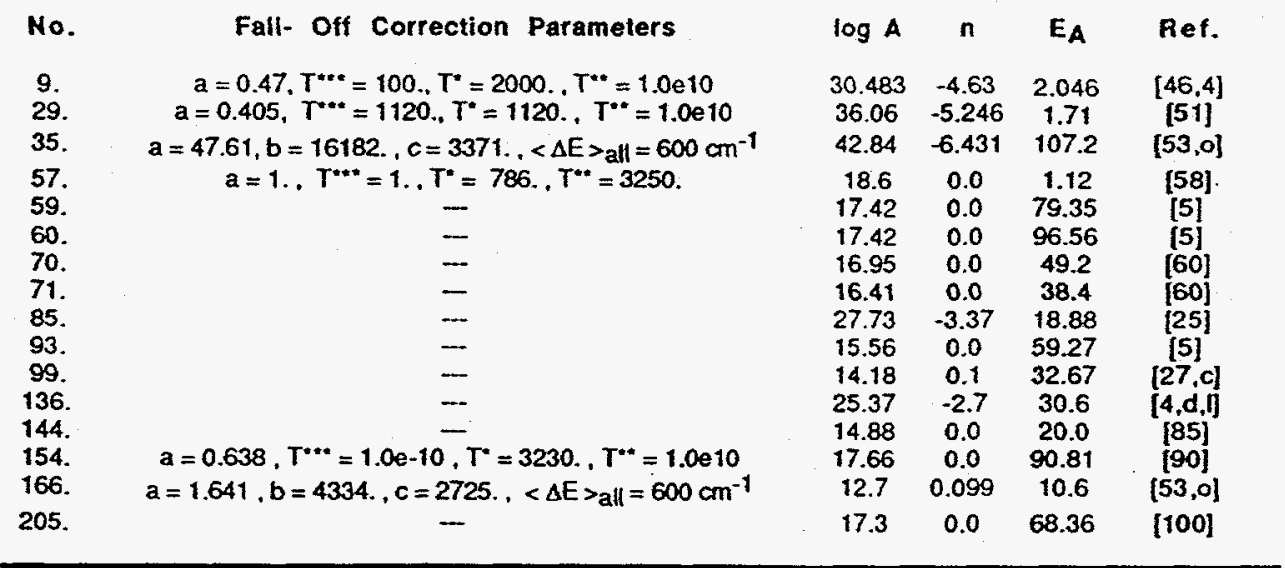

$\left[\mathrm{M}^{\#}\right]=21.0\left[\mathrm{H}_{2} \mathrm{O}\right]+3.8\left[\mathrm{CO}_{2}\right]+16.0\left[\mathrm{CH}_{4}\right]+2.5\left[\mathrm{H}_{2}\right]+1.9[\mathrm{CO}]$

$\left[\mathrm{M}^{*}\right]=16.0\left[\mathrm{H}_{2} \mathrm{O}\right]+3.8\left[\mathrm{CO}_{2}\right]+16.0\left[\mathrm{CH}_{4}\right]+2.5\left[\mathrm{H}_{2}\right]+1.9[\mathrm{CO}]$

$\left[\mathrm{M}^{* *}\right]=10.0\left[\mathrm{H}_{2} \mathrm{O}\right]+2.0\left[\mathrm{O}_{2}\right]+2.0\left[\mathrm{~N}_{2}\right]+2.0\left[\mathrm{H}_{2}\right]$

† All pressure corrected reactions have the high pressure rate expression listed in the full mechanism. The low pressure rate expression is listed below along with its associated fall - off correction parameters.

(a)Fall - off reaction in the Troe form:

$F_{\text {cent }}=(1-a) \exp \left(-T / T^{* * *}\right)+a \exp \left(-T / T^{*}\right)+\exp \left(-T^{* *} / T\right)$

(b) Fitted expression based on Ref. [101-108]

(c)Fall - off reaction in the SRI form: $F^{S C}=a \exp (-b / T)+\exp (-T / c)$

(d) Adjusted

(e)See Text

(O)Fall - off reaction in the Lindemann - Hinshelwood form

(g)QRRK calculated rate expression at one atmosphere, $M=N 2$.

(h) Estimate

(i) Estimate based on $\mathrm{CH} 3 \mathrm{CHO}+\mathrm{X}(\mathrm{X}=\mathrm{OH}, \mathrm{O}, \mathrm{HO} 2, \mathrm{CH} 3)$ reaction.

(j) The pre-exponential frequency factor is estimated by analogy to $\mathrm{CH} 2 \mathrm{O}+\mathrm{O} 2$

reaction. Activation energy based on $\mathrm{CHOCHO} / \mathrm{O} 2 / \mathrm{N} 2$ study of Ref. [109].

(k)Fitted expression based on Ref. [110 - 116].

(1) This Study [23].

(m)Fitted expression based on Ref. [117 - 119]

(n)Fitted expression based on Ref. $[96,120-122]$.

(o) Used Troe relation [123], $\beta_{c} /\left(1-\beta_{c} 0.5\right)=-\left\langle\Delta E>_{\text {all }} / F_{E k B T}\right.$, to obtain $\beta_{c}$ from $\left\langle\Delta E>_{\text {all }}\right.$. Transition state frequencies obtained from [53]. Fitted expressions at one atmosphere are $\mathrm{k}_{35}=10^{51.319} \mathrm{~T}^{-10.60} \mathrm{Exp}(-115.575 \mathrm{kcal} / \mathrm{RT}) \mathrm{sec}^{-1}$ and $k_{166}=10^{14.919} \mathrm{~T}^{-0.50} \operatorname{Exp}(-13.045 \mathrm{kcal} / \mathrm{mol}) \mathrm{cm}^{3} / \mathrm{mol}-\mathrm{sec}$ with $M_{\text {羙 }}=\mathrm{N} 2$. 
Table 3

Thermodynamic Properties

at 1 Atmosphere Standard State

\begin{tabular}{|c|c|c|c|}
\hline Species & $\begin{array}{l}\mathrm{DH}_{\mathrm{f}}(298 \mathrm{~K}) \\
(\mathrm{kcal} / \mathrm{mol})\end{array}$ & $\begin{array}{l}S^{\circ}(298 \mathrm{~K}) \\
(\mathrm{cal} / \mathrm{mol})\end{array}$ & $\mathrm{C}_{\mathrm{p}}(\mathrm{T})$ Calculation \\
\hline $\mathrm{HO} 2$ & $3.8[124]$ & $54.7[21]$ & Kee et al. [21] \\
\hline $\begin{array}{c}\mathrm{C} 2 \mathrm{H} 4 \mathrm{O} 2 \mathrm{H} \\
. \mathrm{CH} 2 \mathrm{CH} 2 \mathrm{OOH} \\
\end{array}$ & $7.3[55]$ & $77.5[55]$ & Bozzelli/Dean [55] \\
\hline $\mathrm{C} 2 \mathrm{H} 4 \mathrm{O}$ & $-12.6[22]$ & $58.0[22]$ & Burcat [22] \\
\hline $\begin{array}{l}\mathrm{C} 2 \mathrm{H} 3 \mathrm{O} \\
{[125]^{\mathrm{b}}}\end{array}$ & 36.0 & 59.8 & $\begin{array}{l}\text { THERM: Primary CH } \\
\text { bond option applied to } \\
\text { C2H4O (Ethylene oxide) } \\
\text { parent: } \\
\text { BDE }=100.6 \mathrm{kcal} / \mathrm{mol}\end{array}$ \\
\hline $\mathrm{C} 2 \mathrm{H} 3$ & $70.4[34]$ & $55.5[21]$ & Kee et al. [21] \\
\hline CHOCHO & $-50.7[126]$ & $66.5[29]$ & $\begin{array}{l}2-\mathrm{CO}(\mathrm{H})(\mathrm{C})^{\mathrm{a}}, 1-\mathrm{OI} \\
\text { Int. Rot. , }[127,128]\end{array}$ \\
\hline $\mathrm{CHOCO}$ & -16.8 & 68.9 & $\begin{array}{c}\text { Parent: } \mathrm{C} \mathrm{H} \mathrm{O} \mathrm{C} \mathrm{H} \mathrm{O} \\
\text { BD group } \mathrm{CCl}^{*} \mathrm{O}[125]^{b}\end{array}$ \\
\hline $\mathrm{CH} 2(\mathrm{~S})$ & $103.9[85]$ & $45.08[21]$ & Kee et al. [21] \\
\hline
\end{tabular}

${ }^{a}$ Assumed $\mathrm{CO}(\mathrm{H})(\mathrm{C})=\mathrm{CO}(\mathrm{H})(\mathrm{CO})$ for $\mathrm{Cp}(\mathrm{T})$ calculation ; ${ }^{\mathrm{b}}$ Estimate $; \mathrm{BD}=\mathrm{Bond}$

Dissociation ; OI $=$ Optical Isomer, Int. Rot. = Internal Rotation, $\mathrm{rd}=$ radical

Chemical kinetic modeling analysis favors the use of the Tully kinetic rate expression over the recent study by Bott and Cohen [129] for this reaction. The non-Arrhenius kinetic expression of Atkinson [56] was used in this study and is based on the experimental results of Tully [17] and Smith [130].

The $\mathrm{O}$-atom reaction with ethylene produces the most prevalent organic intermediates as determined by this study. The Thornton measurements for formaldehyde, methane and methanol exhibited about $1 \%$ maximum yield from the initial ethylene concentration in the 1153 to $1203 \mathrm{~K}$ temperature range. Eventhough formaldehyde is primarily formed from the vinyl reaction with $\mathrm{O} 2$, the $\mathrm{C} 2 \mathrm{H} 4+\mathrm{O}$-atom reaction does lead to formaldehyde production via methyl radical oxidation. Methane and methanol are formed from the methyl radical produced from $\mathrm{C} 2 \mathrm{H} 4+\mathrm{O}$-atom. 
The kinetic rate expression used in this study for the reaction $\mathrm{C} 2 \mathrm{H} 4+$ $\mathrm{O} \rightarrow$ Products was taken from Klemm et al. [57]. Their results are represented by an. Arrhenius expression for the 1052 - 2000K temperature range. This study used the product channels for the $\mathrm{C} 2 \mathrm{H} 4+$ $O$ reaction as suggested by Dagaut et al. [16] and are represented by equations (51) and (52). This study applied a branching ratio value of $\mathrm{k}_{51 / \mathrm{k} 52}=2.3$ to the two product channels in accord with Schmoltner et al. [131].

The $\mathrm{H}$-atom abstraction reaction by $\mathrm{H}$-atom, $\mathrm{C} 2 \mathrm{H} 4+\mathrm{H}=\mathrm{C} 2 \mathrm{H} 3+\mathrm{H} 2$ (53), consumes only a small fraction of the ethylene, though it is one of the primary hydrocarbon reactions for the formation of $\mathrm{H} 2$. The rate expression used was based on the fitted expression of Westbrook et al. [9].

Vinyl Radical Kinetics:

The kinetics of the $\mathrm{C} 2 \mathrm{H} 3+\mathrm{O} 2$ reactions were initially taken from the theoretical analysis work of Westmoreland. Westmoreland [30] proposed that the reaction structure of vinyl and $\mathrm{O} 2$ undergoes multiple isomerizations of chemically activated isomers resulting in several reaction paths and thermal products. The resulting major product channels from the Westmoreland Bimolecular Quantum-RRK study are the reactions shown below for the $300-2000 \mathrm{~K}$ temperature range.

$$
\begin{aligned}
& \mathrm{C} 2 \mathrm{H} 3+\mathrm{O} 2=\mathrm{C} 2 \mathrm{H} 3 \mathrm{O} 2 \\
& \mathrm{C} 2 \mathrm{H} 3+\mathrm{O} 2=\mathrm{CH} 2 \mathrm{O}+\mathrm{HCO} \\
& \mathrm{C} 2 \mathrm{H} 3+\mathrm{O} 2=\mathrm{CHOCHO}+\mathrm{H} \\
& \mathrm{C} 2 \mathrm{H} 3+\mathrm{O} 2=\mathrm{C} 2 \mathrm{H} 2+\mathrm{HO}_{2}
\end{aligned}
$$

This study has examined these product channels and their influence on the combustion intermediates. Formaldehyde is the primary intermediate produced by way of Baldwin and Walkers' [40] proposed mechanism of $\mathrm{C} 2 \mathrm{H} 3$ and $\mathrm{O} 2$ addition, cyclization and beta-scissions to 
make $\mathrm{CH} 2 \mathrm{O}$ and $\mathrm{HCO}$. This reaction channel has the lowest energy barrier to products and subsequently it is favored over internal H-atom abstraction followed by decomposition to $\mathrm{C} 2 \mathrm{H} 2$ plus $\mathrm{HO} 2$ and the channel leading to $\mathrm{CHOCHO}$ and $\mathrm{H}$-atom. The association reaction, $\mathrm{C} 2 \mathrm{H} 3+\mathrm{O} 2=$ $\mathrm{C} 2 \mathrm{H} 3 \mathrm{O} 2$, reverts back to reactants for the conditions examined in this study and is not a major vinyl consuming route.

The rate expressions for the major vinyl radical plus molecular oxygen reaction channels were calculated by Westmoreland with a vinyl radical heat of formation value of $70.4 \mathrm{kcal} / \mathrm{mol}$. The Westmoreland $\mathrm{C} 2 \mathrm{H} 3+\mathrm{O} 2$ product sets, rate expressions and the $\mathrm{C} 2 \mathrm{H} 3$ heat of formation value were implemented into the model.

The Rao and Skinner rate expression [27] for vinyl decomposition to acetylene plus $\mathrm{H}$-atom was pressure corrected using the high pressure rate expression for the reverse reaction by Organ (as cited by Mackie [132]) and the thermodynamic equilibrium rate based on the 70.4 $\mathrm{kcal} / \mathrm{mol}$ vinyl radical heat of formation value. Pressure correction was performed by using the Lindemann-Hinshelwood method.

Modeling efforts with the Westmoreland $\mathrm{C} 2 \mathrm{H} 3+\mathrm{O} 2$ products and rate expressions proved to be unsuccessful. The ethylene consumption and $\mathrm{CH} 4, \mathrm{CH} 3 \mathrm{OH}, \mathrm{C} 2 \mathrm{H} 6, \mathrm{CO}$ and $\mathrm{CO} 2$ profiles were well underpredicted. The $\mathrm{CH} 2 \mathrm{O}$ and $\mathrm{H} 2$ profiles were well predicted and acetylene profile showed a five fold overprediction for all temperatures. The acetylene predicted profile was very sensitive to the rate constant choice for $\mathrm{C} 2 \mathrm{H} 3+\mathrm{O} 2=$ $\mathrm{C} 2 \mathrm{H} 2+\mathrm{HO} 2$ and the pronounced nature of fall-off for the $\mathrm{C} 2 \mathrm{H} 3+\mathrm{O} 2=$ $\mathrm{CH} 2 \mathrm{O}+\mathrm{HCO}$ reaction, while insensitive to vinyl decomposition. A factor of four reduction to the Westmoreland rate expression for the $\mathrm{C} 2 \mathrm{H} 3+\mathrm{O} 2$ $=\mathrm{C} 2 \mathrm{H} 2+\mathrm{HO} 2$ product channel was tried while keeping all other reactions fixed and acetylene predictions improved to within a factor two for the temperature range studied. If the Westmoreland fall-off correction to $\mathrm{C} 2 \mathrm{H} 3+\mathrm{O} 2=\mathrm{CH} 2 \mathrm{O}+\mathrm{HCO}$ was not taken into consideration, e.g. chemical kinetic modeling were to implement the Slagle et al. measured rate expression of $4.0 \times 10^{12} \exp (125 / \mathrm{T}) \mathrm{cm}^{3} / \mathrm{mol}-\mathrm{sec}$ for the 
$\mathrm{C} 2 \mathrm{H} 3+\mathrm{O} 2=\mathrm{CH} 2 \mathrm{O}+\mathrm{HCO}$ reaction, then a rate constant value of approximately $1.25 \times 10^{11} \mathrm{~cm}^{3} / \mathrm{mol}-\mathrm{sec}$ for $\mathrm{C} 2 \mathrm{H} 3+\mathrm{O} 2=\mathrm{C} 2 \mathrm{H} 2+\mathrm{HO} 2$ would be required for accurate acetylene profiling. This latter value concurs with the Tsang and Hampson recommendation and agrees to within a factor of two with the Westmoreland predicted rate expression. Thus, if matching the recommendation of Tsang/Hampson or Westmoreland for the $\mathrm{C} 2 \mathrm{H} 3+\mathrm{O} 2=\mathrm{C} 2 \mathrm{H} 2+\mathrm{HO} 2$ reaction is considered desireable then chemical kinetic modeling analysis indicates that fall-off for the overall $\mathrm{C} 2 \mathrm{H} 3+\mathrm{O} 2 \rightarrow$ Products reaction should be less pronounced than indicated by Westmoreland.

Careful chemical kinetic rate adjustment was then made to those reactions exhibiting rate controlling behavior as determined from reaction-pathway analysis or show significant sensitivity to the ethylene oxidation calculations as determined from the sensitivity analysis. These adjustments were performed while retaining the Westmoreland $\mathrm{C} 2 \mathrm{H} 3+\mathrm{O} 2$ product channels and rate expressions in the chemical kinetic model. The reactions listed below had its respective rate expression altered for the purpose of achieving reasonable agreement with the Thornton data set.

$$
\begin{aligned}
& \mathrm{H}+\mathrm{O} 2+\mathrm{M}=\mathrm{HO} 2+\mathrm{M} \\
& \mathrm{H}+\mathrm{O} 2=\mathrm{OH}+\mathrm{O} \\
& \mathrm{HCO}+\mathrm{M}=\mathrm{H}+\mathrm{CO}+\mathrm{M} \\
& \mathrm{HCO}+\mathrm{O} 2=\mathrm{CO}+\mathrm{HO} 2 \\
& \mathrm{OH}+\mathrm{HO} 2=\mathrm{H} 2 \mathrm{O}+\mathrm{O} 2 \\
& \mathrm{C} 2 \mathrm{H} 4+\mathrm{OH}=\mathrm{C} 2 \mathrm{H} 3+\mathrm{H} 2 \mathrm{O}
\end{aligned}
$$

No satisfactory result was achieved in adjusting these rate expressions. The typical problem encountered was poor profiling of the ethylene, $\mathrm{CO}, \mathrm{CO} 2$ and the aggregate concentration of methane plus methanol for the full temperature range. Also, simultaneous agreement with the ethylene data set of Thornton and for the methane and ethane data sets of Dagaut to within a factor of two for all measured species could not be achieved by careful chemical kinetic rate adjustments of the aforementioned reactions in the presently compiled model. 
Chemical kinetic rate adjustment consideration was then applied to $\mathrm{C} 2 \mathrm{H} 3(+\mathrm{M})=\mathrm{C} 2 \mathrm{H} 2+\mathrm{H}(+\mathrm{M})$. The vinyl decomposition reaction rate adjustment to the levels prescribed in the WP and DCB reaction sets allowed good agreement to be achieved against the Thornton data set for all measured species but acetylene at temperatures less than $1200 \mathrm{~K}$. No agreement could be achieved for temperatures in excess of $1200 \mathrm{~K}$. Furthermore, simultaneous modeling of the Dagaut methane and ethane data sets showed reasonable agreement with the measured profiles for the parent hydrocarbon, the intermediates and by-product $\mathrm{CO} 2$ formed. Computer modeling studies determined that altering the vinyl decomposition or oxidation kinetic rates does not have a significant impact on the methane and ethane oxidation kinetics.

If a high vinyl decomposition chemical kinetic rate, on the order of the DCB prescribed rate, is considered acceptable then a reevaluation of the vinyl decomposition process is in order. The high production rate of acetylene resulting from the vinyl decomposition pathway would rule out the $\mathrm{C} 2 \mathrm{H} 3+\mathrm{O} 2 \rightarrow \mathrm{C} 2 \mathrm{H} 2+\mathrm{HO}_{2}$ as a contributing route to acetylene production for the conditions of this study. The alternative consideration of implementing the revised KWKW vinyl decomposition kinetic rate expression lowers the acetylene and $\mathrm{H}$-atom production rate. The vinyl radical then becomes exclusively consumed by $\mathrm{O} 2$, and the production loss of $\mathrm{H}$-atom (from $\mathrm{C} 2 \mathrm{H} 3 \rightarrow \mathrm{C} 2 \mathrm{H} 2+\mathrm{H}$ ) seriously compromises any modeling agreement with the Thornton data set.

This study increased the chemical kinetic rate to $\mathrm{C} 2 \mathrm{H} 3+\mathrm{O} 2 \rightarrow \mathrm{CHOCHO}$ $+\mathrm{H}$ than previously prescribed by Westmoreland. No reasonable agreement could be achieved toward obtaining an adequate match to the Thornton data set based on this adjusted reaction rate. The kinetic rate limitations imposed by the Gutman et al. experimental studies involving the reaction $\mathrm{C} 2 \mathrm{H} 3+\mathrm{O} 2 \rightarrow$ Products hampered the extent of rate enhancement to this reaction.

Modeling efforts indicate that the $\mathrm{C} 2 \mathrm{H} 3+\mathrm{O} 2$ product channel must produce a reactive free radical in order to attain agreement with the 
Thornton data set. Bozzelli and Dean [30], using the BimolecularQuantumRRK treatment to the $\mathrm{C} 2 \mathrm{H} 3+\mathrm{O} 2$ reaction, suggested a potential vinyl oxidation sequence of $\mathrm{O} 2$ addition to the vinyl followed by an O-atom splitting off

$$
\mathrm{C} 2 \mathrm{H} 3+\mathrm{O} 2 \leftrightarrow \mathrm{C} 2 \mathrm{H} 3 \mathrm{OO} . \leftrightarrow . \mathrm{CH} 2 \mathrm{CHO}+\mathrm{O}
$$

or simply,

$$
\mathrm{C} 2 \mathrm{H} 3+\mathrm{O} 2 \rightarrow . \mathrm{CH} 2 \mathrm{CHO}(\mathrm{C} 2 \mathrm{H} 3 \mathrm{O} \cdot)+\mathrm{O}
$$

They believe that the vinoxy and $\mathrm{O}$-atom product channel is enhanced by the resonance stabilization of the vinoxy which makes this channel more accessible than the analogous channel (ethoxy plus O-atom) in the ethyl plus $\mathrm{O} 2$ reaction system. Their analysis of the $\mathrm{C} 2 \mathrm{H} 3+\mathrm{O} 2$ reaction favors the vinoxy and $\mathrm{O}$-atom product channel for temperatures greater than $900 \mathrm{~K}$. This study initially used the Bimolecular-QRRK predicted $\mathrm{C} 2 \mathrm{H} 3+\mathrm{O} 2=\mathrm{CH} 2 \mathrm{CHO}+\mathrm{O}$ rate expression of $1.191 \mathrm{E} 14 \mathrm{~T}^{-0.611} \exp (-2646 / \mathrm{T})$ $\mathrm{cm}^{3} / \mathrm{mol}-\mathrm{sec}$ [67] based on the arguments presented by Bozzelli and Dean for the viability of this reaction. This kinetic rate was subsequently adjusted upward to $7.0 \mathrm{E} 14 \mathrm{~T}^{-0.611} \exp (-2646 / \mathrm{T}) \mathrm{cm}^{3} / \mathrm{mol}$ sec for complete agreement with the Thornton measured speciation profiles. This rate expression differs by more than two orders of magnitude higher than the Westmoreland prediction [30] and lies a factor of three lower than the Bozzelli and Dean prediction [31] for the conditions of this study.

An explanation for the large difference between the Westmoreland and the Bozzelli/Dean predictions for the vinoxy and $\mathrm{O}$-atom product channel lies in the suitable choice for the high pressure preexponential frequency factor, $A_{\infty}, f_{w d}$, and activation energy barrier, $\mathrm{E}_{\mathrm{act}, \infty}$,fwd, for the reaction $\mathrm{C} 2 \mathrm{H} 3 \mathrm{OO} . \rightarrow . \mathrm{CH} 2 \mathrm{CHO}+\mathrm{O}$. Westmoreland assumed a tight transition state structure for the $\mathrm{O}$-atom addition to the oxygen of the $\mathrm{C}=\mathrm{O}$ bond in. $\mathrm{CH} 2 \mathrm{CHO}$. He calculated a $\mathrm{A}_{\infty}$, rev of $2 \times 10^{10}$ $\mathrm{cm}^{3} \mathrm{~mol}^{-1} \mathrm{sec}^{-1}$ with microscopic reversibility yielding $\mathrm{A}_{\infty}, \mathrm{fwd}=$ $2.1 \times 10^{12} \mathrm{~cm}^{3} \mathrm{~mol}^{-1} \mathrm{sec}^{-1}$. Bozzelli and Dean used the analogy of $\mathrm{O}$-atom 
addition to ethylene to obtain the reverse $\mathrm{A}$-factor for $\mathrm{O}$-atom addition to the oxygen of the carbonyl bond in . $\mathrm{CH} 2 \mathrm{CHO}$. Their analogy yielded a

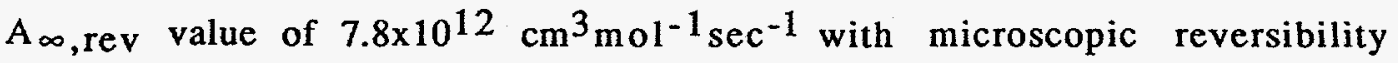
yielding $A_{\infty}, \mathrm{fwd}=3.3 \times 10^{14} \mathrm{~cm}^{3} \mathrm{~mol}^{-1} \mathrm{sec}^{-1}$. The three orders of magnitude difference (at this study's conditions) between the Westmoreland and Bozzelli/Dean QRRK predicted kinetics for $\mathrm{C} 2 \mathrm{H} 3+\mathrm{O} 2=$ $. \mathrm{CH} 2 \mathrm{CHO}+\mathrm{O}$ is primarily due to the $\mathrm{A}_{\infty}$, rev determination. The large difference in $A_{\infty}$, fwd (or $A_{\infty}$, rev ) indicates a problem of interpreting the structure of the chemically activated and resonantly stabilized 2oxo-ethyl - O atom/ethenyloxy - $\mathrm{O}$ atom ( $\mathrm{C} 2 \mathrm{H} 3 \mathrm{O}-\mathrm{-O})$ complex. There is also a $\sim 8.0 \mathrm{kcal} / \mathrm{mol}$ energy barrier difference between the two studies regarding the choice of the $\mathrm{E}_{\mathrm{act}, \infty}$, fwd for the $\mathrm{C} 2 \mathrm{H} 3 \mathrm{OO}$. $\rightarrow \mathrm{CH} 2 \mathrm{CHO}+\mathrm{O}$ reaction or $E_{a c t, \infty, r e v}$ for the addition of O-atom to the oxygen of the carbonyl bond in . $\mathrm{CH} 2 \mathrm{CHO}$. This energy barrier difference accounts for the remaining disagreement in the predicted kinetics for $\mathrm{C} 2 \mathrm{H} 3+\mathrm{O} 2=$ . $\mathrm{CH} 2 \mathrm{CHO}+\mathrm{O}$ between Westmoreland and Bozzelli/Dean. The difference in barrier heights between the two studies is critical for the predicted $\mathrm{C} 2 \mathrm{H} 3+\mathrm{O} 2=. \mathrm{CH} 2 \mathrm{CHO}+\mathrm{O}$ kinetics at low temperatures, whereupon we refer the reader to Bozzelli/Dean's work. The most important difference that can be concluded for high temperature combustion is the choice of the high pressure A-factor for the $\mathrm{C} 2 \mathrm{H} 3 \mathrm{OO} . \leftrightarrow . \mathrm{CH} 2 \mathrm{CHO}+\mathrm{O}$ reaction.

Modeling efforts readily support the $\mathrm{C} 2 \mathrm{H} 3+\mathrm{O} 2$ channel leading to $\mathrm{CH} 2 \mathrm{CHO}+\mathrm{O}$-atom as an extremely important reaction in the ethylene oxidation process. This result indicates that the Bozzelli/Dean intrepretation of the $\mathrm{C} 2 \mathrm{H} 3+\mathrm{O} 2=\mathrm{CH} 2 \mathrm{CHO}+\mathrm{O}$ reaction proceeding through a looser transistion state than perceived by Westmoreland is correct as determined by this modeling study. The $\mathrm{C} 2 \mathrm{H} 3+\mathrm{O} 2=\mathrm{CH} 2 \mathrm{CHO}+$ O-atom reaction followed by $\mathrm{CH} 2 \mathrm{CHO}=\mathrm{CH} 2 \mathrm{CO}+\mathrm{H}$ is a very potent chain branching process involved in ethylene oxidation. The additional O-atom source other than from $\mathrm{H}+\mathrm{O} 2=\mathrm{OH}+\mathrm{O}$ allows for rapid ethylene oxidation by $\mathrm{O}$-atom. The reactive $\mathrm{H}$-atom produced from vinoxy decomposition and from $\mathrm{C} 2 \mathrm{H} 4+\mathrm{O}$-atom reactions can also further the oxidation of the ethylene. The subsequent $\mathrm{C} 2 \mathrm{H} 4+\mathrm{O}$-atom reactions have 
the ability to produce methyl radical which eventually forms methane, methanol, and ethane. Figures 4 and 5 illustrate the agreement between the modelled and experimental measurements for the loss of ethylene and the reaction intermediates' formed due to the inclusion of reaction (96).

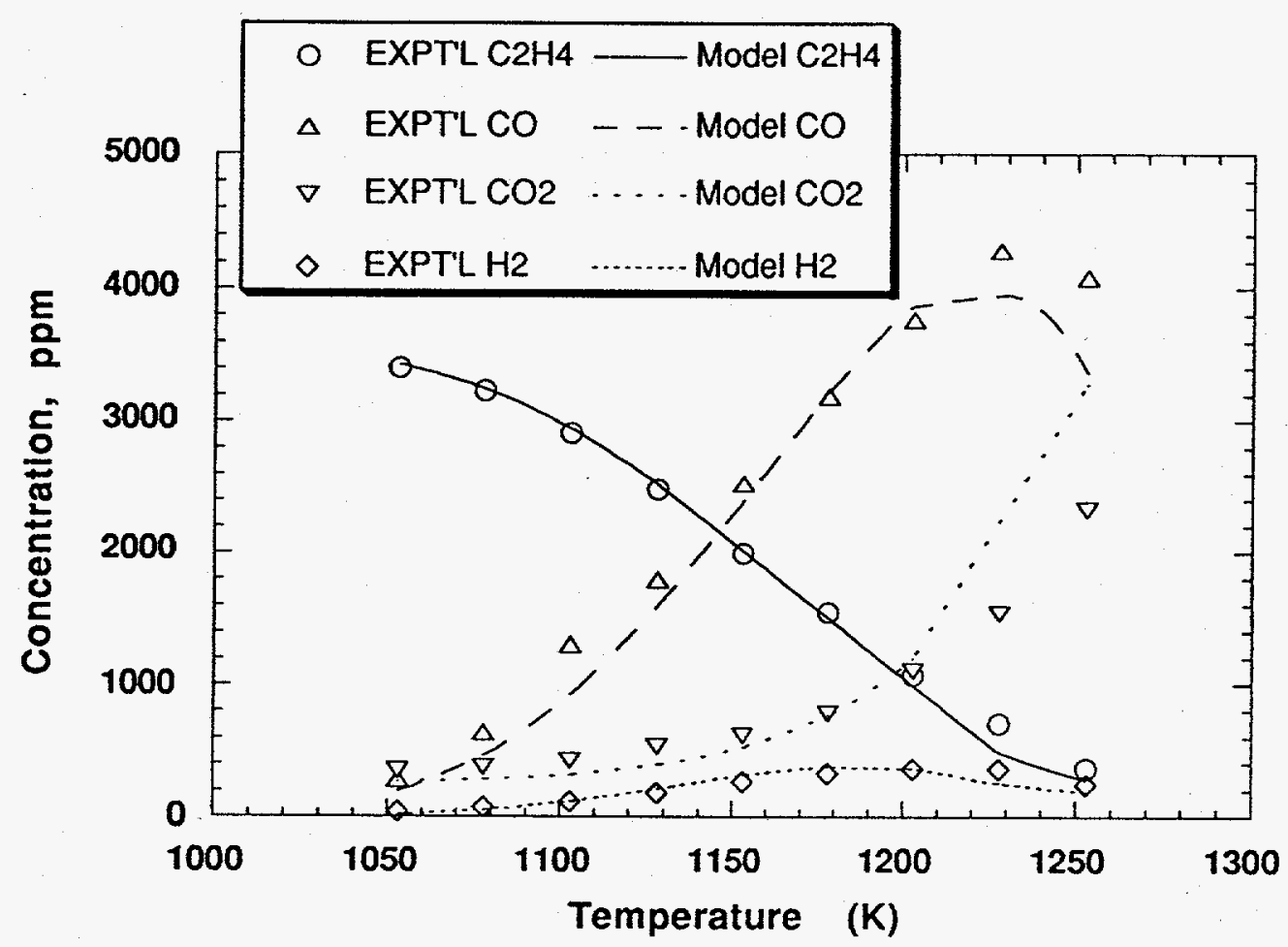

Figure 4: Comparison of predicted and experimental concentration profiles of $\mathrm{C} 2 \mathrm{H} 4, \mathrm{CO}, \mathrm{CO} 2$ and $\mathrm{H} 2$ in the well-stirred reactor for ethylene oxidation: experimental (open symbols), from Thornton; predicted curves from the compiled ethylene oxidation model. 


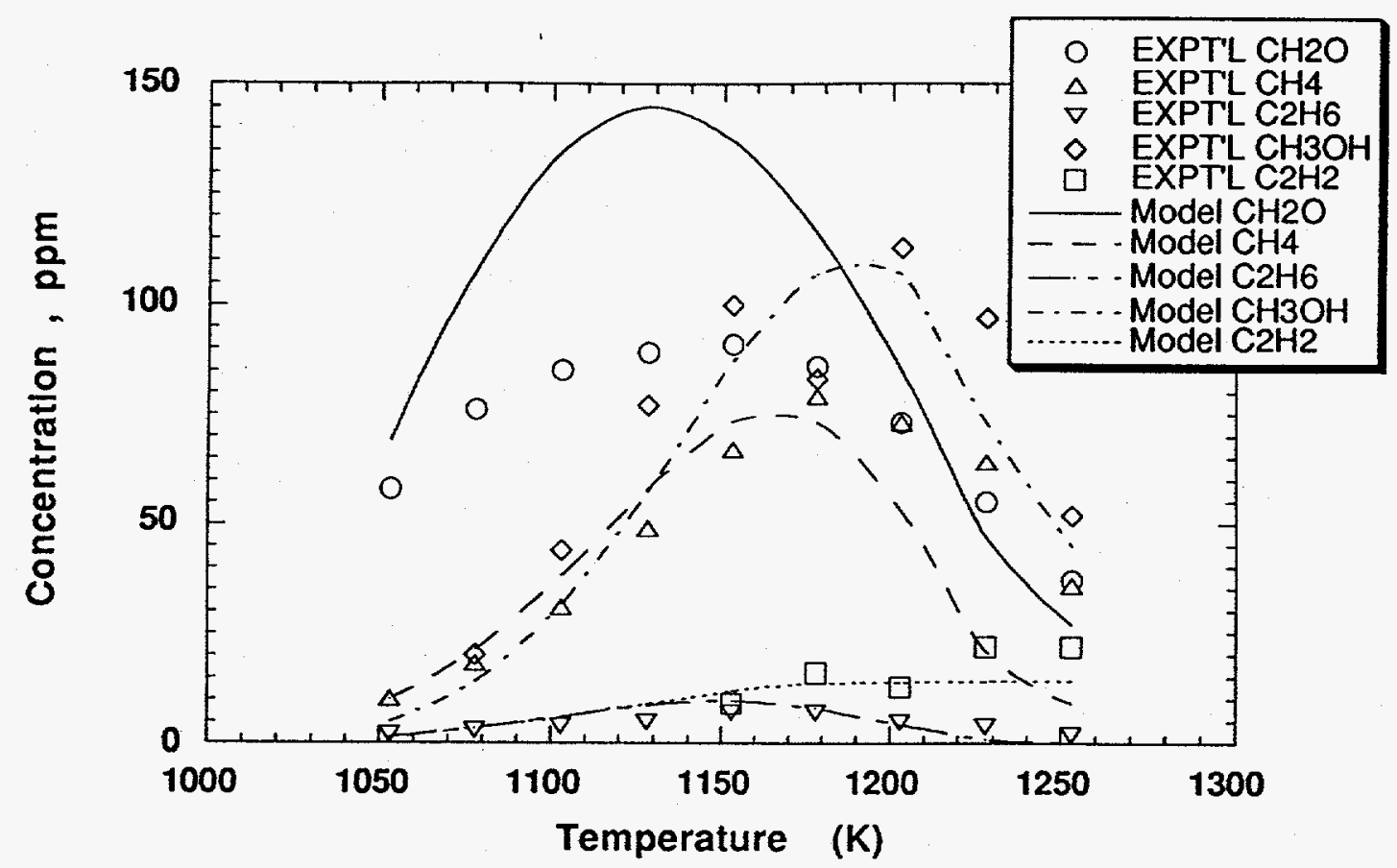

Figure 5: Comparison of predicted and experimental concentration profiles of $\mathrm{CH} 2 \mathrm{O}, \mathrm{CH} 4, \mathrm{CH} 3 \mathrm{OH}, \mathrm{C} 2 \mathrm{H} 6$ and $\mathrm{C} 2 \mathrm{H} 2$ in the well-stirred reactor for ethylene oxidation: experimental (open symbols), from Thornton; predicted curves from the compiled ethylene oxidation model.

Modeling analysis is indeterminate toward obtaining the proper individual kinetic rates for the various $\mathrm{C} 2 \mathrm{H} 3+\mathrm{O} 2$ product pathways since vinyl radical is consumed principally by 02 . However, sensitivity to the modeled predictions is strongly exhibited by the branching ratios selected. If the $\mathrm{C} 2 \mathrm{H} 3+\mathrm{O} 2=\mathrm{CH} 2 \mathrm{O}+\mathrm{HCO}$ reaction exhibits fall-off at high temperatures similar to the predicted kinetics of Westmoreland, then the branching ratios for the product sets, $\mathrm{CH} 2 \mathrm{O}+\mathrm{HCO}: \mathrm{CH} 2 \mathrm{CHO}+\mathrm{O}: \mathrm{C} 2 \mathrm{H} 2+$ $\mathrm{HO} 2$, are $1.0: 1.21: 0.052$ at $1053 \mathrm{~K}$ and $1.0: 2.47: 0.080$ at $1253 \mathrm{~K}$ [Table 2 kinetics]. If the Slagle et al. [29] measured kinetic rate were used for the $\mathrm{C} 2 \mathrm{H} 3+\mathrm{O} 2=\mathrm{CH} 2 \mathrm{O}+\mathrm{HCO}$ reaction, hence no fall-off is exhibited for this reaction, then the branching ratios for the aforementioned product sets would be $1.0: 1.19: 0.067$ at $1053 \mathrm{~K}$ and $1.0: 1.63: 0.068$ at $1253 \mathrm{~K}$ (where the 
kinetic rate of $\mathrm{C} 2 \mathrm{H} 3+\mathrm{O} 2=. \mathrm{CH} 2 \mathrm{CHO}+\mathrm{O}$ would be $4.62 \mathrm{E} 15 \mathrm{~T}^{-0.611} \exp (-$ $2646 / \mathrm{T}) \mathrm{cm}^{3} / \mathrm{mol}-\mathrm{sec}$ and $\mathrm{C} 2 \mathrm{H} 3+\mathrm{O} 2=\mathrm{C} 2 \mathrm{H} 2+\mathrm{HO} 2$ would be $3.00 \mathrm{E} 11$ $\mathrm{cm}^{3} / \mathrm{mol}-\mathrm{sec}$ ). The domination of the $\mathrm{C} 2 \mathrm{H} 3+\mathrm{O} 2=\mathrm{CH} 2 \mathrm{CHO}+\mathrm{O}$ pathway is expected to occur around $1000 \mathrm{~K}$ for one atmosphere pressure.

\section{Remaining Reaction Intermediates Kinetics:}

Acetylene primarily reacts with $\mathrm{O}$-atom to form $\mathrm{CH} 2+\mathrm{CO}$, reaction (106), and $\mathrm{HCCO}+\mathrm{H}$, reaction (107). The product channels, rate expression and branching ratio of $\mathrm{K}_{106} / \mathrm{K}_{107}=0.7$ involving $\mathrm{C} 2 \mathrm{H} 2+\mathrm{O} Æ$ Products were taken from Michael and Wagner [69]. The product channels and rate expressions for $\mathrm{C} 2 \mathrm{H} 2+\mathrm{OH} \rightarrow$ Products, i.e. reactions (108), (109), (110) and (111), were taken from Miller and Melius [70].

Formaldehyde primarily produced by $\mathrm{C} 2 \mathrm{H} 3+\mathrm{O} 2 \rightarrow \mathrm{CH} 2 \mathrm{O}+\mathrm{HCO}$ is consumed by $\mathrm{OH}, \mathrm{O}, \mathrm{H}$ radicals to make $\mathrm{HCO}$. Formyl radicals do not reach large concentrations relative to formaldehyde and are quickly oxidized or decomposed to produce carbon monoxide.

The primary route to methyl radical formation is from $\mathrm{O}$-atom attack on the ethylene. The methyl radical produced is the precursor species to methane, methanol, and ethane formation as observed by Thornton. The reactions leading to methane, methanol and ethane formation are:

$$
\begin{aligned}
& \mathrm{CH} 3+\mathrm{HO} 2=\mathrm{CH} 4+\mathrm{O} 2 \\
& \mathrm{CH} 3+\mathrm{H}+\mathrm{M}=\mathrm{CH} 4+\mathrm{M} \\
& \mathrm{CH} 3+\mathrm{C} 2 \mathrm{H} 4=\mathrm{CH} 4+\mathrm{C} 2 \mathrm{H} 3 \\
& \mathrm{CH} 3+\mathrm{OH}=\mathrm{CH} 3 \mathrm{OH} \\
& \mathrm{CH} 3+\mathrm{CH} 3=\mathrm{C} 2 \mathrm{H} 6
\end{aligned}
$$


Carbon monoxide is primarily formed from the destruction reactions involving the formyl radical. Carbon monoxide is then principally converted to carbon dioxide by $\mathrm{CO}+\mathrm{OH} \rightarrow \mathrm{CO} 2+\mathrm{H}$. Carbon dioxide is primarily formed from carbon monoxide oxidization and minor production from $\mathrm{CH} 2+\mathrm{O} 2 \rightarrow \mathrm{CO} 2+\mathrm{H} 2$ and $\mathrm{HCCO}+\mathrm{O} 2 \rightarrow \mathrm{CO} 2+\mathrm{CO}+\mathrm{H}$.

\section{CHEMICAL KINETIC MODEL ANALYSIS AND VALIDATION}

The 1050K - 1200K Temperature Range

The computational analysis performed with the compiled ethylene oxidation model supports the earlier conclusion of WP and DCB that for the $1050 \mathrm{~K}$ - $1200 \mathrm{~K}$ temperature range the reactor behaves in a wellstirred mode. Furthermore, very good agreement is found between the model's predictions and the experimental measurements for the loss of ethylene and the reaction intermediate concentrations formed as indicated in figures 3 and 4 . Reaction pathway analysis was performed at $1153 \mathrm{~K}$ using the presently compiled model in order to identify the principal and secondary ethylene oxidation pathways.

The principal oxidation pathway was determined to be the following:

$$
\begin{aligned}
& \mathrm{C} 2 \mathrm{H} 4+\mathrm{OH}=\mathrm{C} 2 \mathrm{H} 3+\mathrm{H} 2 \mathrm{O} \\
& \text { (1) } \mathrm{C} 2 \mathrm{H} 3+\mathrm{O} 2=\mathrm{CH} 2 \mathrm{CHO}+\mathrm{O} \\
& \text { (2) } \mathrm{C} 2 \mathrm{H} 4+\mathrm{O}=\mathrm{CH} 3+\mathrm{HCO} \\
& \text { (3) } \mathrm{C} 2 \mathrm{H} 4+\mathrm{O}=\mathrm{CH} 2+\mathrm{HCO}+\mathrm{H} \\
& \text { (1) } \mathrm{CH} 2 \mathrm{CHO}+\mathrm{M}=\mathrm{CH} 2 \mathrm{CO}+\mathrm{H}+\mathrm{M} \\
& \mathrm{CH} 2 \mathrm{CO}+\mathrm{OH}=\mathrm{CH} 2 \mathrm{OH}+\mathrm{CO} \\
& \mathrm{CH} 2 \mathrm{CO}+\mathrm{OH}=\mathrm{HCCO}+\mathrm{H} 2 \mathrm{O} \\
& \mathrm{CH} 2 \mathrm{OH}+\mathrm{O} 2=\mathrm{CH} 2 \mathrm{O}+\mathrm{HO} 2 \\
& \mathrm{HCCO}+\mathrm{O} 2=\mathrm{CO} 2+\mathrm{CO}+\mathrm{OH} \\
& \mathrm{CH} 2 \mathrm{O}+\mathrm{X}=\mathrm{HCO}+\mathrm{XH} \quad \mathrm{X}=\mathrm{OH}, \mathrm{O}, \mathrm{H}
\end{aligned}
$$




$$
\begin{aligned}
& \mathrm{HCO}+\mathrm{O} 2=\mathrm{CO}+\mathrm{HO} 2 \\
& \mathrm{HCO}+\mathrm{M}=\mathrm{H}+\mathrm{CO}+\mathrm{M}
\end{aligned}
$$

$$
\begin{aligned}
& \mathrm{CH} 3+\mathrm{OH}(+\mathrm{M})=\mathrm{CH} 3 \mathrm{OH}(+\mathrm{M}) \\
& \mathrm{CH} 3 \mathrm{OH}+\mathrm{OH}=(\mathrm{CH} 3 \mathrm{O} \text { or } \mathrm{CH} 2 \mathrm{OH})+\mathrm{H} 2 \mathrm{O} \\
& \mathrm{CH} 3+\mathrm{HO} 2=\mathrm{CH} 3 \mathrm{O}+\mathrm{OH} \\
& \mathrm{CH} 3 \mathrm{O}(+\mathrm{M})=\mathrm{CH} 2 \mathrm{O}+\mathrm{H}(+\mathrm{M}) \\
& \mathrm{CH} 2 \mathrm{OH}+\mathrm{O} 2=\mathrm{CH} 2 \mathrm{O}+\mathrm{HO} 2
\end{aligned}
$$

(3) $\mathrm{CH} 2+\mathrm{O} 2 \rightarrow \mathrm{CO}, \mathrm{H} 2 \mathrm{O}, \mathrm{CO} 2, \mathrm{H} 2$ Products

$$
\begin{aligned}
& \mathrm{CO}+\mathrm{OH}=\mathrm{CO} 2+\mathrm{H} \\
& \mathrm{H}+\mathrm{O} 2=\mathrm{OH}+\mathrm{O} \\
& \mathrm{H}+\mathrm{O} 2+\mathrm{M}=\mathrm{HO} 2+\mathrm{M} \\
& \mathrm{OH}+\mathrm{HO} 2=\mathrm{H} 2 \mathrm{O}+\mathrm{O} 2
\end{aligned}
$$

The $\mathrm{C} 2 \mathrm{H} 3+\mathrm{O} 2=\mathrm{CH} 2 \mathrm{CHO}+\mathrm{O}$ reaction followed by $\mathrm{CH} 2 \mathrm{CHO}=\mathrm{CH} 2 \mathrm{CO}+\mathrm{H}$ is a very potent chain branching process for ethylene oxidation. This pathway allows for the formation of $\mathrm{O}$-atom, $\mathrm{H}$-atom, and ketene species. It is essential for promoting the $\mathrm{O}$-atom attack on the ethylene in steps (2) and (3) and providing a source of free radicals for the further ethylene consumption. The $\mathrm{O}$-atom formed from step (1) will preferentially react with the ethylene to form methyl and formyl radicals or methylene, formyl radical and $\mathrm{H}$-atom. The methyl radical is principally consumed by reactions involving $\mathrm{HO} 2$ and $\mathrm{OH}$ radicals. These reactions are $\mathrm{CH} 3+\mathrm{HO} 2=$ $\mathrm{CH} 3 \mathrm{O}+\mathrm{OH}$ and $\mathrm{CH} 3+\mathrm{OH}(+\mathrm{M})=\mathrm{CH} 3 \mathrm{OH}+(+\mathrm{M})$. The methylene primarily reacts with $\mathrm{O} 2$ to form $\mathrm{CO} 2, \mathrm{CO}, \mathrm{H} 2 \mathrm{O}$, and $\mathrm{H} 2$ products. The oxidized ethylene contribution from steps (1 - 3) creates formyl radicals which either decompose to produce $\mathrm{H}$-atoms or oxidize to form $\mathrm{HO} 2$ radicals. The formed $\mathrm{H}$-atom will add to $\mathrm{O} 2$ to make $\mathrm{HO} 2$ radical, but for the experimental conditions of this study, the important $\mathrm{HO} 2$ producing pathway is $\mathrm{HCO}+\mathrm{O} 2=$ $\mathrm{CO}+\mathrm{HO} 2$. The $\mathrm{HO} 2$ radical is primarily removed by $\mathrm{HO} 2+\mathrm{HO} 2=\mathrm{H} 2 \mathrm{O} 2+\mathrm{O} 2$, $\mathrm{HO} 2+\mathrm{OH}=\mathrm{H} 2 \mathrm{O}+\mathrm{O} 2$, and $\mathrm{H}+\mathrm{HO} 2=\mathrm{OH}+\mathrm{OH}$. 
The secondary oxidation pathway was determined to be the following:

$$
\begin{aligned}
& \mathrm{C} 2 \mathrm{H} 4+\mathrm{OH}=\mathrm{C} 2 \mathrm{H} 3+\mathrm{H} 2 \mathrm{O} \\
& \mathrm{C} 2 \mathrm{H} 3+\mathrm{O} 2=\mathrm{CH} 2 \mathrm{O}+\mathrm{HCO} \\
& \mathrm{CH} 2 \mathrm{O}+\mathrm{X}=\mathrm{HCO}+\mathrm{XH} \mathrm{X}=\mathrm{OH}, \mathrm{O}, \mathrm{H} \\
& \text { (1) } \mathrm{HCO}+\mathrm{M}=\mathrm{H}+\mathrm{CO}+\mathrm{M} \\
& \text { (2) } \mathrm{HCO}+\mathrm{O} 2=\mathrm{CO}+\mathrm{HO} 2
\end{aligned}
$$

Net Overall Reaction: (1) $\mathrm{C} 2 \mathrm{H} 4+2 \mathrm{OH}+\mathrm{O} 2 \rightarrow 2 \mathrm{CO}+2 \mathrm{H} 2 \mathrm{O}+2 \mathrm{H}$

$$
\mathrm{C} 2 \mathrm{H} 4+2 \mathrm{OH}+3 \mathrm{O} 2 \rightarrow 2 \mathrm{CO}+2 \mathrm{H} 2 \mathrm{O}+2 \mathrm{HO} 2
$$

The net overall reaction indicates that the secondary oxidation pathway can produce either reactive $\mathrm{H}$-atoms or unreactive $\mathrm{HO} 2$ radicals. The formed $\mathrm{H}$-atoms is primarily consumed by $\mathrm{O} 2(25 \%$ at $1053 \mathrm{~K}$ to $52 \%$ at $1253 \mathrm{~K}$ ) to make $\mathrm{OH}$ and $\mathrm{O}$-atom which futhers ethylene oxidation. The HO2 radical is not active in consuming ethylene at these conditions and prefers to self react or react with $\mathrm{OH}$ and $\mathrm{H}$-atom thereby consuming free radicals and inhibiting ethylene oxidation. The dominance of either the $\mathrm{H}$-atom or $\mathrm{HO} 2$ production pathway is strictly determined by the nature of the formyl radical destruction. For the lower temperatures of this study, formyl radical is primarily removed by step (2). Temperatures greater than $1200 \mathrm{~K}$, formyl radical is consumed equally by steps (1) and (2).

\section{Temperatures Greater Than $1200 \mathrm{~K}$}

The good agreement represented by the ethylene oxidation model predictions against the Thornton measurements in the $1200-1250 \mathrm{~K}$ temperature range differs substantially from the WP and DCB reaction set predictions. The underlying difference in the numerical predictions is due to the kinetics and product pathways involving the vinyl radical and the fundamental role the reaction intermediates play in moderating the $\mathrm{OH}$ concentration within the well-stirred reactor. 
Previously, Westbrook and Pitz explained the observed disagreement between their computed results and the Thornton measurements in the 1200 - $1250 \mathrm{~K}$ temperature range as being caused by the prescence of turbulent species fluctuations within the reactor and ruled out the possibility that a mechanistic change could explain the Thornton measurements in this temperature regime. This explanation was later supported by the chemical kinetic computational investigation of Dagaut/Cathonnet/Boettner.

The presently compiled ethylene oxidation model supports the theory that abundant formation of $\mathrm{HO} 2, \mathrm{CH} 2 \mathrm{O}, \mathrm{CO}$, and $\mathrm{CH} 3$ competes with $\mathrm{C} 2 \mathrm{H} 4$ for $\mathrm{OH}$ at the highest temperatures of the data set. This results in reaction (50) experiencing a reduced share of $\mathrm{OH}$ concentration needed for further ethylene consumption as exhibited in Table 4. The competition for $\mathrm{OH}$ at the higher temperatures would explain why significant amounts of ethylene remain and why it is possible to maintain reasonable spatial homogeneity within the reactor for as high as $1253 \mathrm{~K}$.

Table 4

Computed $\mathrm{OH}$ Normalized Rate of Production Percent Contribution at $1153 \mathrm{~K}$ and $1228 \mathrm{~K}$

\begin{tabular}{|c|c|c|}
\hline Reaction & $1153 \mathrm{~K}$ & $1228 \mathrm{~K}$ \\
\hline $\mathrm{HO} 2+\mathrm{OH}=\mathrm{H} 2 \mathrm{O}+\mathrm{O} 2$ & $21 \%$ & $27 \%$ \\
$\mathrm{H} 2+\mathrm{OH}=\mathrm{H} 2 \mathrm{O}+\mathrm{H}$ & 2 & 4 \\
$\mathrm{H} 2 \mathrm{O} 2+\mathrm{OH}=\mathrm{HO} 2+\mathrm{H} 2 \mathrm{O}$ & 4 & - \\
$\mathrm{CO}+\mathrm{OH}=\mathrm{CO} 2+\mathrm{H}$ & 4 & 15 \\
$\mathrm{C} 2 \mathrm{H} 4+\mathrm{OH}=\mathrm{C} 2 \mathrm{H} 3+\mathrm{H} 2 \mathrm{O}$ & 23 & 14 \\
$\mathrm{CH} 2 \mathrm{OO}+\mathrm{OH}=\mathrm{CH} 2 \mathrm{OH}+\mathrm{CO}$ & 12 & 8 \\
$\mathrm{CH} 2 \mathrm{O}+\mathrm{OH}=\mathrm{HCO}+\mathrm{H} 2 \mathrm{O}$ & 23 & 18 \\
$\mathrm{CH} 3+\mathrm{OH}=\mathrm{CH} 3 \mathrm{OH}$ & 6 & 6 \\
$\mathrm{CH} 3 \mathrm{OH}+\mathrm{OH}=\mathrm{CH} 3 \mathrm{O}+\mathrm{H} 2 \mathrm{O}$ & - & 2 \\
$\mathrm{CH} 3 \mathrm{OH}+\mathrm{OH}=\mathrm{CH} 2 \mathrm{OH}+\mathrm{H} 2 \mathrm{O}$ & - & 2 \\
\hline
\end{tabular}

This study can not refute the likelihood that poor mixing at the microscale level may exist at temperatures greater than $1200 \mathrm{~K}$. However, the WP and DCB poor mixing conclusion is based on their usage of fairly high rates of vinyl dissociation which promotes excessive chain branching at the higher temperatures. In particular, the coupling of acetylene 
production and the build-up of $\mathrm{O}$-atom tosufficient concentrations at the high temperature end of the data set unleashes a potent chain branching process. This is exemplified by the following reaction sequence,

$$
\begin{gathered}
\mathrm{C} 2 \mathrm{H} 4+\mathrm{OH}=\mathrm{C} 2 \mathrm{H} 3+\mathrm{H} 2 \mathrm{O} \\
\mathrm{C} 2 \mathrm{H} 3=\mathrm{C} 2 \mathrm{H} 2+\mathrm{H} \\
\mathrm{H}+\mathrm{O} 2=\mathrm{OH}+\mathrm{O} \\
\mathrm{C} 2 \mathrm{H} 4+\mathrm{O}=\mathrm{CH} 3 \mathrm{CO}+\mathrm{H} \text { (WP only) } \\
\mathrm{C} 2 \mathrm{H} 4+\mathrm{O}=\mathrm{CH} 3+\mathrm{HCO} \text { (DCB only) } \\
\mathrm{C} 2 \mathrm{H} 4+\mathrm{O} \rightarrow \mathrm{CH} 2+\mathrm{HCO}+\mathrm{H} \text { (DCB only) } \\
\mathrm{C} 2 \mathrm{H} 2+\mathrm{O}=\mathrm{HCCO}+\mathrm{H} \\
\mathrm{C} 2 \mathrm{H} 2+\mathrm{O}=\mathrm{CH} 2+\mathrm{CO} \\
\mathrm{HCCO}+\mathrm{O} 2=\mathrm{CO}+\mathrm{CO}+\mathrm{OH}(\mathrm{DCB} \text { only }) \\
\mathrm{HCCO}+\mathrm{H}=\mathrm{CH} 2+\mathrm{CO} \\
\mathrm{HCCO}+\mathrm{O}=\mathrm{CO}+\mathrm{CO}+\mathrm{H} \\
\mathrm{HCCO}+\mathrm{OH}=\mathrm{HCO}+\mathrm{CO}+\mathrm{H}(\mathrm{DCB} \text { only }) \\
\mathrm{CH} 2+\mathrm{O} 2=\mathrm{CO} 2+\mathrm{H}+\mathrm{H} \\
\mathrm{CH} 2+\mathrm{O} 2=\mathrm{CO}+\mathrm{OH}+\mathrm{H} \\
\mathrm{CO}+\mathrm{OH}=\mathrm{CO} 2+\mathrm{H} \\
\mathrm{HCO} \rightarrow \mathrm{H} \text { or } \mathrm{HO} 2 \mathrm{radicals}
\end{gathered}
$$

which produces a plethora of reactive free radicals that eventually consumes all the ethylene at the higher temperatures of the dataset as indicated earlier by WP and DCB.

In conclusion, the principal difference between this study's ethylene oxidation model and the WP and DCB mechanisms is the importance of vinyl oxidation kinetics, in particular, the $\mathrm{C} 2 \mathrm{H} 3+\mathrm{O} 2=$ $\mathrm{CH} 2 \mathrm{CHO}+\mathrm{O}$ reaction. The resulting reaction sequence from vinyl oxidation allows for rapid chain branching and favors the formation of stable products such as formaldehyde, methane and methanol. Acetylene kinetics does not play a significant role in the ethylene oxidation process as noted in the Thornton data set and supported by the 
presently compiled ethylene oxidation mechanism. This finding differs from the proposed ethylene oxidation mechanism of Westbrook/Pitz and Dagaut/Cathonnet/Boettner which favors considerable participation of acetylene kinetics in the ethylene oxidation process.

This study supports the viability of the $\mathrm{C} 2 \mathrm{H} 3+\mathrm{O} 2=\mathrm{CH} 2 \mathrm{CHO}+\mathrm{O}$ reaction. Implementation of this reaction should be considered for future high temperature combustion chemistry modeling studies.

\section{SUMMARY}

The reported mechanisms of WP and DCB were used to understand the important features of the ethylene oxidation chemistry. The DCB mechanism showed agreement to the ethylene profile at the lower temperatures of the data set and for an extended temperature range than WP. This was due to the heavy reliance by DCB on the vinyl decomposition to $\mathrm{C} 2 \mathrm{H} 2+\mathbf{H}$ as the predominant vinyl consumption reaction.

The reaction intermediates predicted by the WP and DCB showed limited agreement in temperature range and for certain measured species. In particular, the predicted versus measured acetylene profile showed gross disagreement, and thereby ruling out vinyl dissociation as the prominent vinyl destruction process.

The inadequacies represented by these mechanisms was the motivation for developing the current ethylene oxidation model.

The ethylene oxidation model developed in the present study addressed the issue of whether or not the vinyl oxidation pathway produces a reactive free radical early in the ethylene oxidation process or if careful chemical kinetic rate adjustment must be made to the most sensitive rate controlling reactions in order to obtain agreement with the ethylene oxidation measurements of Thornton. 
The model made use of the Westmoreland rate parameters for the important product sets involving the $\mathrm{C} 2 \mathrm{H} 3+\mathrm{O} 2$ reaction. The modeling results involving the ethylene and the reaction intermediates formed proved unsuccessful with the Westmoreland $\mathrm{C} 2 \mathrm{H} 3+\mathrm{O} 2$ predictions. Careful chemical kinetic rate adjustment made to the most sensitive rate controlling reactions proved unsuccessful when trying to achieve agreement with the ethylene, methane and ethane oxidation data sets.

The $\mathrm{C} 2 \mathrm{H} 3+\mathrm{O} 2$ pathway to $\mathrm{CH} 2 \mathrm{CHO}+\mathrm{O}$-atom proved to be a very important reaction in the ethylene oxidative pyrolysis. The very potent chain branching sequence of $\mathrm{C} 2 \mathrm{H} 3+\mathrm{O} 2 \rightarrow \mathrm{CH} 2 \mathrm{CHO}+\mathrm{O}$-atom followed by $\mathrm{CH} 2 \mathrm{CHO}(+\mathrm{M}) \rightarrow \mathrm{CH} 2 \mathrm{CO}+\mathrm{H}(+\mathrm{M})$ is needed to accurately model the Thornton ethylene data set.

Reaction pathway analysis was utilized to determine the principal and secondary ethylene oxidation routes. The principal oxidation pathway was determined to be the following:

(1) $\mathrm{C} 2 \mathrm{H} 4+\mathrm{OH}=\mathrm{C} 2 \mathrm{H} 3+\mathrm{H} 2 \mathrm{O}$

$\mathrm{C} 2 \mathrm{H} 3+\mathrm{O}_{2}=\mathrm{CH} 2 \mathrm{CHO}+\mathrm{O}$

(2) $\mathrm{C} 2 \mathrm{H} 4+\mathrm{O}=\mathrm{CH} 3+\mathrm{HCO}$

(3) $\mathrm{C} 2 \mathrm{H} 4+\mathrm{O}=\mathrm{CH} 2+\mathrm{HCO}+\mathrm{H}$

(1) $\mathrm{CH} 2 \mathrm{CHO}+\mathrm{M}=\mathrm{CH} 2 \mathrm{CO}+\mathrm{H}+\mathrm{M}$

$\mathrm{CH} 2 \mathrm{CO}+\mathrm{OH}=\mathrm{CH} 2 \mathrm{OH}+\mathrm{CO}$

$\mathrm{CH} 2 \mathrm{CO}+\mathrm{OH}=\mathrm{HCCO}+\mathrm{H} 2 \mathrm{O}$

$\mathrm{CH} 2 \mathrm{OH}+\mathrm{O} 2=\mathrm{CH} 2 \mathrm{O}+\mathrm{HO} 2$

$\mathrm{HCCO}+\mathrm{O} 2=\mathrm{CO} 2+\mathrm{CO}+\mathrm{OH}$

$\mathrm{CH} 2 \mathrm{O}+\mathrm{X}=\mathrm{HCO}+\mathrm{XH} \quad \mathrm{X}=\mathrm{OH}, \mathrm{O}, \mathrm{H}$

$\mathrm{HCO} \rightarrow \mathrm{H}$ or $\mathrm{HO} 2$ radicals

(2) $\mathrm{CH} 3+\mathrm{OH}(+\mathrm{M})=\mathrm{CH} 3 \mathrm{OH}(+\mathrm{M})$

$\mathrm{CH} 3 \mathrm{OH}+\mathrm{OH}=(\mathrm{CH} 3 \mathrm{O}$ or $\mathrm{CH} 2 \mathrm{OH})+\mathrm{H} 2 \mathrm{O}$

$\mathrm{CH} 3+\mathrm{HO}_{2}=\mathrm{CH} 3 \mathrm{O}+\mathrm{OH}$

$\mathrm{CH} 3 \mathrm{O}(+\mathrm{M})=\mathrm{CH} 2 \mathrm{O}+\mathrm{H}(+\mathrm{M})$

$\mathrm{CH} 3+\mathrm{HO}_{2}=\mathrm{CH} 4+\mathrm{O} 2$

(3) $\mathrm{CH} 2+\mathrm{O} 2 \rightarrow \mathrm{CO}, \mathrm{H} 2 \mathrm{O}, \mathrm{CO} 2, \mathrm{H} 2$ Products

$$
\begin{aligned}
& \mathrm{CO}+\mathrm{OH}=\mathrm{CO} 2+\mathrm{H} \\
& \mathrm{H}+\mathrm{O} 2=\mathrm{OH}+\mathrm{O} \\
& \mathrm{H}+\mathrm{O} 2+\mathrm{M}=\mathrm{HO} 2+\mathrm{M} \\
& \mathrm{OH}+\mathrm{HO} 2=\mathrm{H} 2 \mathrm{O}+\mathrm{O} 2
\end{aligned}
$$


The secondary oxidation pathway was determined to be the following:

$$
\begin{gathered}
\mathrm{C} 2 \mathrm{H} 4+\mathrm{OH}=\mathrm{C} 2 \mathrm{H} 3+\mathrm{H} 2 \mathrm{O} \\
\mathrm{C} 2 \mathrm{H} 3+\mathrm{O} 2=\mathrm{CH} 2 \mathrm{O}+\mathrm{HCO} \\
\mathrm{CH} 2 \mathrm{O}+\mathrm{X}=\mathrm{HCO}+\mathrm{XH} \mathrm{X}=\mathrm{OH}, \mathrm{O}, \mathrm{H} \\
\mathrm{HCO}+\mathrm{M}=\mathrm{H}+\mathrm{CO}+\mathrm{M} \\
\mathrm{HCO}+\mathrm{O} 2=\mathrm{CO}+\mathrm{HO} 2 \\
\mathrm{CO}+\mathrm{OH}=\mathrm{CO} 2+\mathrm{H}
\end{gathered}
$$

Good agreement between the compiled model's predictions and the experimental measurements were attained for the 1053 to $1253 \mathrm{~K}$ temperature range. The favorable chemical kinetic modeling results for temperatures greater than $1200 \mathrm{~K}$ suggested that the rate of mixing was sufficiently rapid to maintain reasonable spatial homogeneity within the reactor for temperatures as high as $1253 \mathrm{~K}$.

The $\mathrm{C} 2 \mathrm{H} 3+\mathrm{O} 2$ reaction to form $\mathrm{CH} 2 \mathrm{CHO}+\mathrm{O}$ proved to play an integral part in the ethylene oxidation process and should be used in future high temperature combustion chemistry modeling studies. 


\section{Bibliography}

1. Thornton, M.M.: PhD Thesis, University of Washington, 1989.

2. Westbrook, C.K.; Thornton, M.M.; Pitz, W.J.; and Malte, P.C.:

Twenty-Second Symposium (International) on Combustion, 863 , The Combustion Institute, 1988.

3. Dagaut, P.; Cathonnet, M.; and Boettner, J.C.:

Int. J. Chem. Kinet., $23,437,(1991)$.

4. Tsang, W.; and Hampson, R.F.: J. Phys. Chem. Ref. Data, 15 , $1095,(1986)$.

5. Warnatz, J.: In Combustion Chemistry; (Gardiner, W.C., Ed.); Chapter 5, Springer-Verlag , 1984.

6. Chakir, A.; Cathonnet, M.; Boettner, J.C.; and Gaillard, F.: Combust. Sci. and Tech., 65, 207, (1989).

7. Pitz,W.J.; Westbrook,C.K.; Proscia,W.M.; and Dryer, F.L.: Twentieth Symposium (International) on Combustion, 831, The Combustion Institute, 1985.

8. Brezinsky, K.: Prog. Energy Combust. Sci., 12, 1, (1986).

9. Westbrook, C.K.; Dryer, F.L.; and Schug, K.P.: Nineteenth Symposium (International) on Combustion, 153, The Combustion Institute, 1982.

10. Westbrook, C.K.: Combust. Flame, 46, 191, (1982).

11. Westbrook, C.K.; Dryer, F.L.; and Schug, K.P.:

Combust. Flame, 52, 299, (1983).

12. Wilk,R.D.; Cernansky,N.P.; Westbrook,C.K.; and Pitz, W.J.:

Twenty-Third Symposium (International) on

Combustion, 203, The Combustion Institute, 1990.

13. Cathonnet, M.; Gaillard, F.; Boettner, J.C.; Cambray, P.; Karmed, D.; and Bellet, J.C.: Twentieth Symposium (International) on Combustion, 819, The Combustion Institute, 1984. 
14. Dagaut, P.; Cathonnet, M.; Gaillard, F.; Boettner, J.C.; Rouan, J.P.; and James, H.: Prog. Astro. and Aeronaut., 105. 377, (1986).

15. Dagaut, P.; Cathonnet, M.; Boettner, J.C.; and Gaillard, F.: Combustion and Flame, 71, 295, (1988).

16. Dagaut, P.; Boettner, J.C.; and Cathonnet, M.: Int. J. Chem. Kinet, 22, 641, (1990).

17. Tully, F.P.: Chem. Phys. Lett., 143, 510, (1988).

18. Thornton, M.M.; Malte, P.C.; and Crittenden, A.L.:

Twenty-First Symposium (International) on Combustion, 979, The Combustion Institute, 1986.

19. Thornton, M.M.; Malte, P.C.; and Crittenden, A.L.: Combust. Sci. Tech., 54, 275, 1987.

20. Glarborg, P.; Kee, R.J.; Grcar, J.F.; and Miller, J.A.: Sandia National Laboratory, Albuquerque, N.M., SAND86-8209, 1986.

21. Kee, R.J.; Rupley, F.M.; and Miller, J.A.: Sandia National Laboratory, Albuquerque, N.M., SAND87-8215B, 1987.

22. Burcat, A.: In Combustion Chemistry; (Gardiner, W.C., Ed.); Chapter 8, Springer-Verlag , 1984.

23. Marinov, N.M.: PhD Thesis, University of Washington, 1993.

24. Garo, A.; Westmoreland, P.R.; Howard, J.R.; and Longwell, J.P.: Combustion and Flame, $72,271,(1988)$.

25. Baulch, D.L.; Cobos, C.J.; Cox, R.A.; Esser, C.; Frank, P.; Just, Th.; Kerr, J.A.; Pilling, M.J.; Troe, J.; Walker, R.W.; and Warnatz, J.: J. Phys. Chem. Ref. Data, 21, 411, (1992).

26. Miller, J.A., and Bowman, C.T.: Prog. Energy Combust. Sci., 15, 287, (1989).

27. Rao, V.S. and Skinner, G.B.: J. Phys. Chem., 92, 6313, (1988).

28. Kiefer, J.H.; Wei, H.C.; Kern, R.D.; and Wu, C.H.: Int. J. Chem. Kinet., 17, 225, (1985). 
29. Slagle, I.R.; Park, J-Y.; Heaven, M.C.; and Gutman, D.:

J. Amer. Chem. Soc., 106, 4356, (1984).

30. Westmoreland, P.R.: Combust. Sci. Tech., 82, 15, (1992).

31. Bozzelli, J.W. and Dean, A.M.: J. Phys. Chem., 27. 4427, (1993).

32. Peck, R.E.; Glarborg, P.; and Johnsson, J.E.:

Combust. Sci. Tech., Z6, 81, (1991).

33. Ayranci, G. and Back, M.H.: Int. J. Chem. Kinet., 15. $83,(1983)$.

34. Shiromaru, H.; Achiba, Y.; Kimura, K.; and Lee, Y.T.:

J. Phys. Chem., 21, 17, (1987).

35. McMillian, D.F. and Golden, D.M.: Annu. Rev. Phys. Chem., 33, 493, (1982).

36. Ervin, K.M.; Gronert, S.; Barlow, S.E.; Gilles, M.K.; Harrison, A.G.; Bierbaum, V.M.; Depuy, C.H.,; Lineberger, W.C.; and Ellison, G.B: J. Am. Chem. Soc., 112, 5750, (1990).

37. Benson, S.W. and Haugen, G.R.: J. Phys. Chem., 71, 1735, (1967).

38. Payne, W.A. and Stief, L.J.: J. Chem. Phys., 64, 1150, (1976).

39. Sugarawa, K.; Okazaki, K.; and Sato, S.:

Bull. Chem. Soc. Jpn., 54, 2878, (1981).

40. Baldwin, R.R., and Walker, R.W.: Eighteenth Symposium (International) on Combustion, 819, The Combustion Institute, 1981.

41. Park, J-Y.; Heaven, M.C.; and Gutman, D.:

Chem. Phys. Letters, 104, 469, (1984).

42. Cooke, D.F. and Williams, A.: Thirteenth Symposium (International) on Combustion, 757, The Combustion Institute, (1971). 
43. Wagner, A.F.; Slagle, I.R.; Sarzynski, D.; and Gutman, D.:

J. Phys. Chem., 44, 1853, (1990).

44. Dagaut, P.; Boettner, J.C.; and Cathonnet, M.:

Combust. Sci. Tech., 77,127, (1991).

45. Cherian,M.A. , Rhodes,P. , Simpson,R.J. and Dixon-Lewis, G.:

Phil. Trans. Roy. Soc. London, A303, 181, (1981).

46. Brouwer, L.; Cobos, C.J.; Troe, J.; Dubai, H.R.; and Crim, F.F.:

J. Chem. Phys., 86, 6171, (1987).

47. Harding, L.B. and Wagner, A.F.: Twenty-Second Symposium (International) on Combustion, 983, The Combustion Institute, 1988.

48. Masten, D.A.; Hanson, R.K.; and Bowman, C.T.:

J. Phys. Chem., 94, 7119, (1990).

49. Hippler, H.; Troe, J.; and Willner, J.: J. Chem. Phys., 93, 1755, (1990).

50. Sutherland,J.W.; Michael,J.V.; Pirraglia,A.N.; Nesbitt,F.L.; and Klemm, R.B.: Twenty-First Symposium (International) on Combustion, 929, The Combustion Institute, 1986.

51. Walter, D.; Grotheer, H.H.; Davies, J.W.; Pilling, M.J.; and Wagner, A.F.: Twenty-Third Symposium (International) on Combustion, 107, The Combustion Institute, 1990.

52. Klemm,R.B.; Sutherland,J.W.; Patterson,P.M.; and Tanzana,T.:

Twenty-Fourth Symposium (International) on Combustion, Poster 53, The Combustion Institute.

53. Stewart, P.H.; Larson, C.W.; and Golden, D.M.: Combustion and Flame, 75, 25, (1989).

54. Slagle I.R.; Sarzyriski, D.; Gutman, D.; Miller, J.A.; and Melius, C.F.: J. Chem. Soc., Faraday Trans. 2, 84, 491, (1988).

55. Bozzelli, J.W. and Dean, A.M.: J. Phys. Chem., 24, 3313, (1990). 
56. Atkinson, R.: J. Phys. Chem. Ref. Data, Monograph No. 1, (1989).

57. Klemm, R.B.; Sutherland, J.W.; Wickramaaratchi, M.A.; and Yarwood, G.: J. Phys. Chem., 94, 3354, (1990).

58. Lightfoot, P.D. and Pilling, M.J.: J. Phys. Chem., 91, 3373, (1987).

59. Roscoe, J.M. and Back, R.A.: J. Phys. Chem., 90, 598, (1986).

60. Saito, K.; Kakumoto, T.; and Murakami, I.:

J. Phys. Chem., 88, 1182, (1984).

61. Becker, K.H.; and Klein, Th.: Proceedings, Fourth European Symposium on the Physico-Chemical Behavior of

Atmospheric Pollutants, 1986; 320, D. Riedel

Publishing Co., Dordrecht, Holland, (1987).

62. Niki, H.; Maker, P.D.; Savage, C.M:; and Breitenbach, L.P.:

Int. J. Chem. Kinet., 17, 547, (1985).

63. Colket, M.B. III; Naegeli, D.W. and Glassman, I.: Sixteenth Symposium (International) on Combustion, 1023, The Combustion Institute, 1977.

64. Vandooren, J. and Van Tiggelen, P.J.: Sixteenth Symposium (International) on Combustion, 1133, The Combustion Institute, 1976.

65. Washida, N.; Hatakeyama, S.; and Tagaki, H.:

J. Chem. Phys., 78, 4533, (1983).

66. Miller, J.A.; Mitchell, R.E.; Smooke, M.D. and Kee, R.J.: Nineteenth Symposium (International) on Combustion, 181, The Combustion Institute, 1982.

67. Cedarbalk, P.; Hughes, K.J.; Pilling, M.J.; and Proudler, V.K.: Twenty-Third Symposium (International) on Combustion, Poster 263, The Combustion Institue, 1990.

68. Westmoreland, P.R.: Private Communication. 
69. Heineman, P.; Hofmann-Sievert, R.; and Hoyermann, K.:

Twenty-First Symposium (International) on Combustion, 865, The Combustion Institute, 1986.

70. Michael, J.V. and Wagner, A.F.: J.Phys. Chem., 94 ,2453, (1990).

71. Miller, J.A. and Melius, C.F.: Twenty-Second Symposium (International) on Combustion, 1031. The Combustion Institute, 1988.

72. Vinckier, C.; Schaekers, M.; and Peeters, J.:

J. Phys. Chem., 89, 508, (1985).

73. Peeters, J.; Schaekers, M.; and Vinckier, C.: J. Phys. Chem., 90. 6552, (1982).

74. Harding, L.B.; Schatz, G.C.; and Chiles, R.A.:

J. Chem. Phys.,76, 5172, (1982).

75. Stephens, J.W.; Hall, J.L.; Solka, H.; Yan, W.; Curl, R.F.; and Glass, G.P.: J. Phys. Chem., 91, 5740, (1987).

76. Hwang, S.; Gardiner, W.C. Jr.; Frenklach, M.; and Hidaka, Y.: Combustion and Flame, 67, 65, (1987).

77. Herron, J.T.: J. Phys. Chem. Ref. Data, 17, 966, (1988).

78. Hochgreb, S.; Yetter, R.A.; and Dryer, F.L.: Twenty-Third Symposium (International) on Combustion, 171, The Combustion Institute, 1990.

79. Choudhury, T.K.; Sanders, W.A.; and Lin, M.C.: J. Chem Soc., Faraday Trans. 2, 85, 801, (1989).

80. Timonen, R.S.; Ratajczak, F.; Gutman, D. and Wagner, A.F.: J.Phys. Chem., 21, 5325, 1987.

81. Batt, L.: Int. Rev. Phys. Chem. 6, 53, 1979.

82. Wantuck, P.J.; Oldenborg, R.C.; Baughcum, S.L.; and Winn, K.R.: J. Phys. Chem., 91, 4653, (1987).

83. Frenklach, M.; Wang, H.; and Rabinowitz, M.J.: Prog. Energy Combust. Sci., 18, 47, (1992). 
84. Wantuck, P.J.; Oldenborg, R.C.; Baughcum, S.L.; and Winn, K.R.:

Chem. Phys. Lett., 138, 548, (1987).

85. Greenhill, P.G.; O'Grady, B.V.; and Gilbert, R.G.:

Aust. J. Chem., 39, 1929, (1980).

86. Grotheer, H.H.; Riekert, G.; Walter, D.; and Just, Th.:

Twenty-Second Symposium (International) on Combustion, 963, The Combustion Institute, 1988.

87. Tsang, W.: J. Phys. Chem. Ref. Data, 81, 2367, (1987).

88. Rabinowitz, M.J.; Sutherland, J.W., Patterson, P.M.; and Klemm, R.B.: J. Phys. Chem., 95, 674, (1991).

89. Baldwin, R.R.; Jones, P.N.; and Walker, R.W.: J. Chem. Soc., Faraday Trans. 2: 84, 199, (1988).

90. Cobos, C.J. and Troe, J.: Zeit. Physik. Chemie Neue Folge, 167, 129, (1990).

91. Grotheer, H.H.; Kelm, S.; Driver, H.S.T.; Hutcheon, R.J.; Lockett, R.D.; and Robertson, G.N.: Ber. Bunsenges.

Phys. Chem., 96, 1360, (1992).

92. Dean, A.M., and Westmoreland, P.R.: Int. J. Chem. Kinet., 19, 207, (1987).

93. Hidaka,Y.; Nakamura,T.; Tanaka,H.; Inami,K.; and Kawano, H.: Int. J. Chem. Kinet., 22, 701, (1990).

94. Glarborg, P; Miller, J.A.; and Kee, R.J.: Combustion and Flame, 65, 177, (1986).

95. Dombrowsky, Ch.; Hwang,S.M.; Rohrig,M.; and Wagner, H. Gg.: Ber. Bunsenges. Phys. Chem., 96,194, (1992).

96. Bohland, T.; Temps, F.; and Wagner, H. Gg.: J. Phys. Chem., 91, 1205, (1987). 
97. Zabarnick, S.; Fleming, J.W.; and Lin, M.C.: Twenty-First Symposium (International) on Combustion, 713, The Combustion Institute, 1986.

98. Choudhury, T.K., and Lin, M.C.: Combust. Sci. Tech., 64, 19, (1989).

99. Cathonnet, M. , Boettner, J.C. and James, H.: J. de Chimie Physique, 79, 475, (1982).

100. Spindler, K.G. and Wagner, H. Gg.:

Ber. Bunsenges. Phys. Chem., 86, 119, (1982).

101. Hippler, H.; Neunaber, $H_{\text {.; }}$ and Troe, J.: Twenty-Fourth Symposium (International) on Combustion, Poster 59, The Combustion Institute, 1992.

102. Keyser, L.: J. Phys. Chem., 84, 1659, (1980).

103. Wine, P.; Semmes, D.; and Ravishankara, A.: J. Chem. Phys., 75, 4390, (1981).

104. Baldwin, R.R., and Mayor, L.: Trans. Faraday Soc., 57, 1578, (1960).

105. Baldwin, R.R., and Bratten, D.: Eighth Symposium (International) on Combustion, 110, The Combustion Institute, (1962).

106. Baldwin, R.R.; Jackson, D.; Walker, R.W.; and Webster, R.J.: Tenth Symposium (International) on Combustion, 423, The Combustion institute, (1965).

107. Kurylo, M.; Murphy, J.; Haller, G.; and Curnett, K.: Int. J. Chem. Kinet., 14, 1149, (1982).

108. Greiner, N.: J. Chem. Phys., 72, 406, (1968).

109. Hay, J.M. and Norrish, R.G.W.: Proc. Roy. Soc. Lond., A288, 1, (1965).

110. Veyret, B. and Lesclaux, R.: J. Phys. Chem., 85, 1918, (1981). 
111. Stief, L.J.; Nesbitt, F.L.; and Gleason, J.F.: Abstract of paper pesented at the International Symposium of Gas Kinetics, Assissi, Italy, September 1990.

112. Tsuboi, T.: Jpn. J. Appl.Phys., 15, 159, (1976).

113. Cherian,M.A.; Rhodes,P.; Simpson,R.J.; and Dixon-Lewis,G.: Eighteenth Symposium (International) Combustion, 385, The Combustion Institute, 1981.

114. Langford, A.O. and Moore, C.B.: J. Chem. Phys., 80, 4211, (1984).

115. Temps, F. and Wagner, H.Gg.: Ber. Bunsenges. Phys. Chem., 88, 410, (1984).

116. Timonen, R.S.; Ratajczak, E.; and Gutman, D.:

J. Phys. Chem., 22, 651, (1988).

117. Bhaskaran, K.A.; Frank, P.; and Just, Th.: Proceedings of the Twelfth Shock Tube Symposium, 503, The Magnes Piere, 1979, Jerusalem.

118. Klatt, M.; Rohrig, M. and Wagner, H.G.:

Ber. Bunsenges. Phys. Chem., 95, 1163, (1991).

119. Grela, M.; Amorebreta, V.T. and Colussi, A.:

J. Phys. Chem., 26, 7013, (1992).

120 Zabarnick, S.; Fleming, J.W.; and Lin, M.C.: J. Chem. Phys, 85, 4373, (1986).

121. Frank, P.; Bhaskaran, K.A.; and Just, Th.: J. Phys. Chem., 20, 2226, (1986).

122. Peeters, J. and Vinckier, C.: Fifteenth Symposium (International) on Combustion, 969, The Combustion Institute, 1974.

123. Troe, J.: J. Chem. Phys., 66, 4758, (1977).

124. Fisher, E.R. and Armentrout, P.B.: J. Phys. Chem., 94, 4396, (1990). 
125. Ritter, E.R. and Bozzelli, J.W.: Int. J. Chem. Kinet., 23, 767, (1991). THERM Code Version 4.21.

126. Fletcher, R.A. and Pilcher, G.: Trans. Faraday Soc., 66, 794, (1970).

127. Benson, S.W.: Thermochemical Kinetics, Second Ed., Wiley, 1976.

128. Orville-Thomas, W.J.: Internal Rotation in Molecules, Wiley, 1974.

129. Bott, J.F. and Cohen, N.: Int. J. Chem. Kinet., 23, 1075, (1991).

130. Smith, G.P.: Int. J. Chem. Kinet., 19, 269, (1987).

131. Schmoltner, A.M.; Chu, P.M.; Brudzynski, R.J.; and Lee, Y.T.: J. Chem. Phys., 21, 6926, (1989).

132. Mackie, J.C.: Catal. Rev. - Sci. Eng.: 33, 169, (1991). 